Article

\title{
Preparation and Characterization of Novel Mixed Periodic Mesoporous Organosilica Nanoparticles
}

\author{
Hao Li ${ }^{1,2}\left(\mathbb{D}\right.$, Laurence Raehm ${ }^{2}$, Clarence Charnay ${ }^{2} \mathbb{D}$, Jean-Olivier Durand ${ }^{2, *(\mathbb{D})}$ and \\ Roser Pleixats $1, *$ (D)
}

1 Department of Chemistry and Centro de Innovación en Química Avanzada (ORFEO-CINQA), Faculty of Sciences, Universitat Autònoma de Barcelona. UAB Campus, C/ dels Til.lers, 08193 Cerdanyola del Vallès, Spain; Hao.Li@uab.cat

2 ICGM, CNRS, ENSCM, University of Montpellier, Case 1701, Place Eugène Bataillon, CEDEX 05, 34095 Montpellier, France; clarence.charnay@umontpellier.fr (C.C.); laurence.raehm@umontpellier.fr (L.R.)

* Correspondence: jean-olivier.durand@umontpellier.fr (J.-O.D.); roser.pleixats@uab.cat (R.P.); Tel.: +34-935812067 (R.P.)

Received: 3 March 2020; Accepted: 26 March 2020; Published: 28 March 2020

check for updates

\begin{abstract}
We report herein the preparation of mixed periodic mesoporous organosilica nanoparticles (E-Pn 75/25 and 90/10 PMO NPs) by sol-gel co-condensation of E-1,2-bis(triethoxysilyl)ethylene ((E)-BTSE or E) with previously synthesized disilylated tert-butyl 3,5-dialkoxybenzoates bearing either sulfide (precursor P1) or carbamate (precursor P2) functionalities in the linker. The syntheses were performed with cetyltrimethylammonium bromide (CTAB) as template in the presence of sodium hydroxide in water at $80^{\circ} \mathrm{C}$. The nanomaterials have been characterized by Transmission Electron Microscopy (TEM), nitrogen-sorption measurements (BET), Dynamic Light Scattering (DLS), zeta-potential, Thermogravimetric Analysis (TGA), FTIR, ${ }^{13} \mathrm{C}$ CP MAS NMR and small angle X-ray diffraction ( $p$-XRD). All the nanomaterials were obtained as mesoporous rodlike-shape nanoparticles. Remarkably, E-Pn 90/10 PMO NPs presented high specific surface areas ranging from 700 to $970 \mathrm{~m}^{2} \mathrm{~g}^{-1}$, comparable or even higher than pure E PMO nanorods. Moreover, XRD analyses showed an organized porosity for E-P1 90/10 PMO NPs typical for a hexagonal 2D symmetry. The other materials showed a worm-like mesoporosity.
\end{abstract}

Keywords: periodic mesoporous organosilica nanoparticles; sol-gel process; disilylated tert-butyl 3,5-dialkoxybenzoates

\section{Introduction}

Periodic mesoporous organosilica materials, at the bulk (PMO) [1-3] and nanoscale (PMO NPs) level, are fundamentally unique because they have several advantages, such as robust porous organic-inorganic framework [4], porous channels [5], tunable pore size organization [6,7], biocompatibility [8], and the highest organic content (nano)materials [9]. Moreover, the functionalization of the inner and outer surface allows the modulation of the properties and enable dispersibility in aqueous or organic solvents [10]. The hydrophilicity/hydrophobicity of the pores is also adjustable [11,12]. The PMO (and PMO NPs) can be degraded when specific functional groups sensitive to redox, acid-base, biochemical, or photochemical reactions are present in the structure of the organic framework [13-15].

The post-modification of the organic fragment is also possible by classical organic chemistry [16,17]. Especially in nanomedicine, properties of PMO NPs such as low hemolytic behavior [18], increased biocompatibility, higher loading capacities, physico-chemical adjustability of the pores [19] and designed biodegradability [20], are particularly important when compared to mesoporous silica nanoparticles. 
PMO and PMO NPs are synthesized from organo-bridged alkoxysilanes by the sol-gel process in the presence of surfactants that act as templates. The incorporation of organic moieties as bridging components, directly and specifically into the pore walls, by using disilylated organosilica precursors, enable the preparation of a new generation of organosilica (nano)materials that combines the advantages of organic and inorganic units while overcoming their intrinsic disadvantages [6,20-23]. If there is only one bridged organosilica precursor, pure PMO NPs can be formed. When two bridged organosilica precursors with different functional groups are used, mixed PMO NPs are obtained with two different functionalities in the framework wall.

Specifically, the synthesis of PMO NPs implies the careful control of a range of conditions, such as the reaction temperature, reaction time, $\mathrm{pH}$, solvent, precursors, optimized reactants ratio, catalyst, mixing method and stirring speed. The experimental conditions control the template-silica interactions, the silica condensation rate, the assembly kinetics and, thus, the nucleation and growth rates. Control over the nanostructure, morphological uniformity, size distribution, porosity and compositions of PMO NPs is quite challenging. Interestingly, different types of PMO NPs can be obtained through templating strategies such as spherical nanoparticles, nanorods, nanofibers, and multipodal NPs [9,23].

Although a broad array of PMO materials have been reported, achieving the nanoscale for such materials is still challenging. The first PMO NPs with a hollow spherical morphology (HPMO NPs) were prepared from 1,2-bis(trimethoxysilyl)ethane by using the FC-4 fluorocarbon surfactant $\left[\mathrm{C}_{3} \mathrm{~F}_{7} \mathrm{O}\left(\mathrm{CFCF}_{3} \mathrm{CF}_{2} \mathrm{O}\right)_{2} \mathrm{CFCF}_{3} \mathrm{CONH}\left(\mathrm{CH}_{2}\right)_{3} \mathrm{~N}^{+}\left(\mathrm{C}_{2} \mathrm{H}_{5}\right)_{2} \mathrm{CH}_{3}, \mathrm{I}^{-}\right]$and $\mathrm{CTAB}$ cationic surfactant as co-structure directing agents [24]. In addition, by changing the ratio of FC-4 to CTAB, the shell thickness of HPMO NPs can be adjusted, and non-hollow PMO microspheres can be obtained by using only CTAB surfactant. The procedure followed to obtain phenylene-bridged PMO nanospheres was based on the use of two templates, namely, poly(ethylene oxide)-poly(DL-lactic acid-co-glycolic acid)-poly(ethylene oxide) triblock copolymer and the FC-4 surfactant [25]. Interestingly, the authors found that these two surfactants were immiscible, the core being templated by the polymer based on poly-lactic acid and the shell by FC-4 [25]. When Pluronic P123 block copolymer alone was used as template (without FC-4), hollow silica nanospheres were obtained instead of the expected PMO material [26]. Later, a variety of PMO nanospheres with different organic bridged linkers, such as methylene, ethylene, ethynylene and phenylene was reported $[18,26,27]$. In 2005, Lu and colleagues were the first to use Pluronic P123 triblock copolymer as a structure-directing agent to control the synthesis of ethylene bridged PMO nanorods [28]. Jaroniec and colleagues then described the use of both 1,2-bis (triethoxysilyl)ethane (BTEE) and 1,4-bis (triethoxysilyl)benzene (BTEB) as organosilica precursors, and Pluronic P123 poly(ethylene oxide)-poly(propylene oxide)-poly(ethylene oxide) (PEO-PPO-PEO) as triblock copolymer template to control the synthesis of ethylene-phenylene wide microrods with coil-like morphology [29]. In 2009, Mohanty and Landskron used the trisilylated octaethoxy-1,3,5-trisilapentane as precursor with P123 surfactant, and PMO nanorods and nanofibers were obtained with an adjustable aspect ratio from 2:1 to 20:1 by varying the concentration of the precursor [30,31]. In 2011, methylene-, ethenylene-, and phenylene-bridged PMO helical fibers were prepared using CTAB and (S)- $\beta$-citronellol as templating agents [32]. The sizes and shapes could be adjusted to obtain nanorods depending on the precursor used. In some research works, CTAB was used as the only template to prepare bridged PMO nanorods [7,11]. Interestingly, Khashab's group reported the first controlled syntheses of para-phenylene-bridged PMO NPs with different morphologies, from nanospheres to nanorods and nanowires, with CTAB as a structure-directing agent by adjusting the organic co-solvents [33]. Multipodal PMO NPs with phenylene-bridged cores and ethenylene-bridged pods were designed by Croissant et al., which were prepared in a CTAB-templated aqueous medium with sodium hydroxide as catalyst through a one-pot two-step method [34]. While the sole condensation of the 1,4-bis(triethoxysilyl)benzene or 1,2-bis-(triethoxysilyl)ethylene precursor afforded phenylene- and ethenylene-bridged PMO nanospheres and nanorods, respectively, the addition of the ethenylene precursor into a solution of freshly prepared phenylene-bridged NPs gave rise to multipodal nano-objects. Interesting reviews on the synthetic strategies for the preparation of PMO 
nanomaterials and their applications in nanomedicine and catalysis have been published [20,23,35-37]. Furthermore, the glutathione-triggered degradability of PMO and related materials by incorporating disulfide bridges in the structure has been comprehensively reviewed [14].

The synthesis of PMO NPs with large and flexible organic bridging groups within the mesoporous wall remains a scientific challenge and the development of new mixed periodic mesoporous organosilica nanoparticles is very relevant, especially for potential new applications in nanomedicine.

Herein, we describe the preparation and full characterization of novel mixed PMO NPs which have been obtained by sol-gel co-condensation of E-1,2-bis(triethoxysilyl)ethylene (BTSE or E) [38] with disilylated tert-butyl 3,5-dialkoxybenzoates bearing either sulfide (precursor P1) or carbamate (precursor P2) functionalities in the linker (Figure 1).<smiles>CCOCCCSCCCCOc1cc(OCCCCSCCC[SiH2]OCC)cc(C(=O)OCC)c1</smiles><smiles>CCO[SiH2]CCCNC(=O)OCCCOc1cc(OCCCOC(=O)NCCC[SiH2]CC)cc(C(=O)OCC)c1</smiles>

Figure 1. Proposed disilylated tert-butyl 3,5-dialkoxybenzoates P1 and $\mathbf{P 2}$ for the preparation of mixed PMO NPs.

\section{Materials and Methods}

\subsection{General Remarks}

The ${ }^{13}$ C-CP-MAS solid state NMR spectra (Les Ullis, France), the BET surface areas (Mérignac, France), Dynamic Light Scattering (DLS, Pessac, France) and Zeta potential values (Orsay, France) were obtained from equipments of University of Montpellier [39]. The ${ }^{1} \mathrm{H}$ - and ${ }^{13} \mathrm{C}-\mathrm{NMR}$ spectroscopy (Bruker Biospin, Rheinstetten, Germany), Mass-Spectrometry (MS, Bruker Biosciences Española, Madrid, Spain), elemental analysis, Infra-Red spectroscopy (IR, Bruker Biosciences Española, Madrid, Spain), Powder X-Ray diffraction (P-XRD, Almelo, Netherlands) and Transmission Electron Microscopy (TEM, JEOL Ltd., Akishima, Tokyo, Japan) were performed at the Universitat Autonoma de Barcelona (UAB) [39].

When necessary, experiments were carried out with Schlenk techniques under standard high vacuum. $\mathrm{CTAB}, \mathrm{NH}_{4} \mathrm{NO}_{3}$, sodium hydroxide, potassium bromide and the required reagents for the preparation of P1 and P2 were purchased from Sigma-Aldrich (Madrid, Spain). Ethanol and acetone were purchased from Fisher Chemicals (Madrid, Spain) and hydrochloric acid from VWR PROLABO (Barcelona, Spain). E PMO NPs were prepared as described [11].

\subsection{Synthesis of $\mathbf{2}$}

In a $250 \mathrm{~mL}$ Schlenk flask under nitrogen atmosphere, but-3-en-1-ol $1(8.0 \mathrm{~mL}, 0.843 \mathrm{~g} / \mathrm{mL}$, $93.50 \mathrm{mmol})$ was added, dissolved in dry dichloromethane $(100 \mathrm{~mL})$ and dry triethylamine $(5.0 \mathrm{~mL}$, $0.73 \mathrm{~g} / \mathrm{mL}, 117 \mathrm{mmol}$ ). Then, the mixture was stirred with cooling (ice bath) over a period of $15 \mathrm{~min}$. After this time, methanesulfonyl chloride $(15.0 \mathrm{~mL}, 1.48 \mathrm{~g} / \mathrm{mL}, 193.80 \mathrm{mmol})$ was added and the reaction mixture was stirred overnight at room temperature under argon atmosphere. Then, $100 \mathrm{~mL}$ of fresh dichloromethane were added to the crude and it was washed first with $\mathrm{HCl} 5 \mathrm{M}(50 \mathrm{~mL} \times 2)$ and then with saturated aqueous solution of $\mathrm{Na}_{2} \mathrm{CO}_{3}(50 \mathrm{~mL} \times 2)$ and brine $(50 \mathrm{~mL} \times 2)$. The organic fraction was dried using anhydrous sodium sulfate and the solvent evaporated under vacuum to afford 
the final product 2 as yellow liquid (13.80 g, 99\% yield) [40]. ${ }^{1} \mathrm{H} \mathrm{NMR}\left(360 \mathrm{MHz}, \mathrm{CDCl}_{3}\right) \delta$ (ppm): $5.84-5.73(\mathrm{~m}, 1 \mathrm{H}), 5.21-5.14(\mathrm{~m}, 2 \mathrm{H}), 4.27(\mathrm{t}, J=7.2 \mathrm{~Hz}, 2 \mathrm{H}), 3.01(\mathrm{~s}, 3 \mathrm{H}), 2.54-2.48(\mathrm{~m}, 2 \mathrm{H})$.

\subsection{Synthesis of $\mathbf{4}$}

To the stirred mixture of 3,5-dihydroxybenzaldehyde $3(1.38 \mathrm{~g}, 10.0 \mathrm{mmol})$ and cesium carbonate $(13.03 \mathrm{~g}, 40.0 \mathrm{mmol})$ in anhydrous DMF $(80 \mathrm{~mL})$ an excess of but-3-en-1-yl methanesulfonate 2 $(4.50 \mathrm{~g}, 30.0 \mathrm{mmol})$ was added. The reaction was stirred at $40{ }^{\circ} \mathrm{C}$ under argon atmosphere until 3,5-dihydroxybenzaldehyde was fully consumed (TLC monitoring, $48 \mathrm{~h}$ ). Then, water was added $(100 \mathrm{~mL})$ and the product was extracted using hexane $(200 \mathrm{~mL} \times 3)$. The organic phase was washed with $1 \mathrm{M} \mathrm{NaOH}$, dried with anhydrous sodium sulfate and the solvent was removed under vacuum to afford the final product 4 as yellow liquid (1.93 g, 78\% yield) [41]. ${ }^{1} \mathrm{H} \mathrm{NMR}\left(360 \mathrm{MHz}, \mathrm{CDCl}_{3}\right) \delta(\mathrm{ppm})$ : $9.89(\mathrm{~s}, 1 \mathrm{H}), 7.00(\mathrm{br} \mathrm{s}, 2 \mathrm{H}), 6.71(\mathrm{br} \mathrm{s}, 1 \mathrm{H}), 5.93-5.84(\mathrm{~m}, 2 \mathrm{H}), 5.20-5.11(\mathrm{~m}, 4 \mathrm{H}), 4.05(\mathrm{t}, J=7.2 \mathrm{~Hz}, 4 \mathrm{H})$, 2.58-2.55 (m, 4H); ${ }^{13} \mathrm{C} \mathrm{NMR}\left(91 \mathrm{MHz}, \mathrm{CDCl}_{3}\right) \delta$ (ppm): 192.0, 160.5, 138.3, 134.1, 117.3, 108.1, 107.8, 67.6, 33.5. IR (film): 3078.9, 2980.0, 2876.7, 1697.7, 1592.4, 1452.6, 1295.6, 1296.0, 1165.9, 1065.9, 1065.1, 843.9, $676.0 \mathrm{~cm}^{-1}$. GC-MS (EI) m/z: $246.2\left[\mathrm{M}^{+}\right], 192.1,163.1,150.1,138.0,121.1,110.0,91.1,75.0,65.1$, 55.1; HRMS (ESI) $\mathrm{m} / \mathrm{z}[\mathrm{M}+\mathrm{Na}]^{+}$calcd for $\mathrm{C}_{15} \mathrm{H}_{18} \mathrm{O}_{3} \mathrm{Na}$ : 269.1148, found: 269.1141.

\subsection{Synthesis of 5}

To a stirred solution of 3,5-bis(but-3-en-1-yloxy)benzaldehyde $4(1.97 \mathrm{~g}, 8.0 \mathrm{mmol})$ in acetonitrile $(12.0 \mathrm{~mL})$ was added a solution of $\mathrm{NaH}_{2} \mathrm{PO}_{4} \cdot 2 \mathrm{H}_{2} \mathrm{O}(374.4 \mathrm{mg}, 2.4 \mathrm{mmol})$ in water $(5.0 \mathrm{~mL})$ and $30 \%$ $\mathrm{H}_{2} \mathrm{O}_{2}(4.0 \mathrm{~mL}, 9.97 \mathrm{~mol} / \mathrm{L}, 40.0 \mathrm{mmol})$. Finally, a solution of $80 \%$ purity $\mathrm{NaClO}_{2}(1.27 \mathrm{~g}, 11.2 \mathrm{mmol})$ in water $(10.0 \mathrm{~mL})$ was added dropwise during $2 \mathrm{~h}$ to the stirred mixture at room temperature. $\mathrm{O}_{2}$ evolved from the solution was monitored until the end of the reaction with a bubbler connected to the flask. A small amount of $\mathrm{Na}_{2} \mathrm{SO}_{3}$ was added to destroy the excess of oxidants. The mixture was acidified with $10 \%$ aqueous $\mathrm{HCl}$, and the product was extracted using ethyl acetate $(60 \mathrm{~mL} \times 3)$. The organic phase was dried with anhydrous sodium sulfate and the solvent was removed under vacuum to afford a residue which was purified by a flash column chromatography through silica gel eluting with hexane/EtOAc $=2: 1$, obtaining the product 5 as a colorless solid $\left(1.78 \mathrm{~g}, 85 \%\right.$ yield) [42]. ${ }^{1} \mathrm{H}$ NMR $\left(360 \mathrm{MHz}, \mathrm{CDCl}_{3}\right) \delta$ (ppm): $7.25(\mathrm{~d}, J=3.6 \mathrm{~Hz}, 2 \mathrm{H}), 6.70(\mathrm{br} \mathrm{s}, 1 \mathrm{H}), 5.94-5.85(\mathrm{~m}, 2 \mathrm{H}), 5.21-5.11(\mathrm{~m}, 4 \mathrm{H})$, $4.05(\mathrm{t}, J=7.2 \mathrm{~Hz}, 4 \mathrm{H}), 2.58-2.53(\mathrm{~m}, 4 \mathrm{H}) ;{ }^{13} \mathrm{C} \mathrm{NMR}\left(91 \mathrm{MHz}, \mathrm{CDCl}_{3}\right) \delta(\mathrm{ppm}): 171.7,160.0,134.2,130.9$, $117.2,108.4,107.6,67.5,33.5$.

\subsection{Synthesis of $\mathbf{6}$}

In a $10 \mathrm{~mL}$ round bottom flask, 3,5-bis(but-3-en-1-yloxy)benzoic acid 5 (1.31 g, $5.0 \mathrm{mmol})$, di-tert-butyl dicarbonate $(2.18 \mathrm{~g}, 10.0 \mathrm{mmol})$ and 4-dimethylaminopyridine $(183.3 \mathrm{mg}, 1.5 \mathrm{mmol})$ were dissolved in tert-butanol $(40 \mathrm{~mL})$. The reaction mixture was stirred at room temperature under argon atmosphere until 3,5-bis(but-3-en-1-yloxy)benzoic acid 5 was fully consumed (TLC monitoring, $48 \mathrm{~h}$ ). Then, the solvent was removed under reduced pressure and the residue was purified by flash column chromatography through silica gel eluting with hexane/AcOEt $=15: 1$, obtaining the pure product 6 as colorless oil (1.38 g, 87\% yield) [43]. ${ }^{1} \mathrm{H}$ NMR $\left(360 \mathrm{MHz}, \mathrm{CDCl}_{3}\right) \delta(\mathrm{ppm}): 7.13(\mathrm{~d}, J=3.6 \mathrm{~Hz}, 2 \mathrm{H}), 6.62$ $(\mathrm{t}, J=3.6 \mathrm{~Hz}, 1 \mathrm{H}), 5.94-5.84(\mathrm{~m}, 2 \mathrm{H}), 5.20-5.10(\mathrm{~m}, 4 \mathrm{H}), 4.03(\mathrm{t}, J=7.2 \mathrm{~Hz}, 4 \mathrm{H}), 2.56-2.53(\mathrm{~m}, 4 \mathrm{H}), 1.58$ (s, 9H); ${ }^{13} \mathrm{C} \mathrm{NMR}\left(91 \mathrm{MHz}, \mathrm{CDCl}_{3}\right) \delta$ (ppm): 165.5, 159.8, 134.3, 133.9, 117.1, 107.8, 105.9, 81.2, 67.4, 33.5, 28.2. IR (film): $3078.7,2977.7,1711.7,1594.3,1445.8,1300.5,1159.9,1049.1,915.6,767.2 \mathrm{~cm}^{-1}$. GC-MS (EI) m/z: $318.2[\mathrm{M}]^{+}, 262.1,245.2,208.1,190.1,166.1,154.0,136.0,55.1 ; \mathrm{HRMS}$ (ESI) m/z [M + Na] ${ }^{+}$calcd for $\mathrm{C}_{19} \mathrm{H}_{26} \mathrm{O}_{4} \mathrm{Na}$ : 341.1723 , found: 341.1707 .

\subsection{Synthesis of $\mathbf{P} \mathbf{1}$}

In a $50 \mathrm{~mL}$ Schlenk tube under nitrogen, tert-butyl 3,5-bis(but-3-en-1-yloxy)benzoate 6 (954.5 mg, $3.0 \mathrm{mmol})$ and 2,2-dimethoxy-1,2-diphenylethanone (DMPA) $(153.8 \mathrm{mg}, 0.6 \mathrm{mmol})$ were dissolved in anhydrous THF (12.0 mL). Then (3-mercaptopropyl)triethoxysilane $(1.5 \mathrm{~g}, 6.3 \mathrm{mmol})$ was added and 
the stirred mixture was irradiated with a UV lamp at $365 \mathrm{~nm}$ under argon atmosphere until tert-butyl 3,5-bis(but-3-en-1-yloxy)benzoate 6 was fully consumed (TLC monitoring, $16 \mathrm{~h}$ ). Then, the solvent was removed under vacuum and the crude product was purified by a flash column chromatography through silica gel eluting with hexane/AcOEt $=10: 1$, obtaining the pure product P1 as colorless oil (1.66 g, 70\% yield) [44]. ${ }^{1} \mathrm{H}$ NMR $\left(360 \mathrm{MHz}, \mathrm{CDCl}_{3}\right) \delta$ (ppm): 7.09 (br s, 2H), 6.58 (br s, $\left.1 \mathrm{H}\right), 3.97$ (t, $J=$ $7.2 \mathrm{~Hz}, 4 \mathrm{H}), 3.82(\mathrm{q}, J=7.2 \mathrm{~Hz}, 12 \mathrm{H}), 2.56$ (apparent q, $J=7.2 \mathrm{~Hz}, 8 \mathrm{H}), 1.88-1.76(\mathrm{~m}, 4 \mathrm{H}), 1.74-1.70(\mathrm{~m}$, $8 \mathrm{H}), 1.57(\mathrm{~s}, 9 \mathrm{H}), 1.21(\mathrm{t}, J=7.2 \mathrm{~Hz}, 18 \mathrm{H}), 0.74(\mathrm{t}, J=7.2 \mathrm{~Hz}, 4 \mathrm{H}) ;{ }^{13} \mathrm{C} \mathrm{NMR}\left(91 \mathrm{MHz}, \mathrm{CDCl}_{3}\right) \delta(\mathrm{ppm})$ : 165.6, 159.9, 133.8, 107.6, 105.7, 81.2, 67.6, 58.4, 35.1, 31.6, 28.4, 28.1, 26.2, 23.2, 18.3, 9.9. IR (film): 2972.2, 2925.1, 1713.7, 1594.6, 1445.3, 1389.1, 1326.1, 1248.8, 1161.9, 1074.2, 956.6, $768.1 \mathrm{~cm}^{-1}$. MS (ESI) m/z: 817.4 [M + Na] $]^{+}, 749.4,647.3,425.3,393.3 ;$ HRMS (ESI) $\mathrm{m} / \mathrm{z}[\mathrm{M}+\mathrm{Na}]^{+}$calcd for $\mathrm{C}_{37} \mathrm{H}_{70} \mathrm{O}_{10} \mathrm{~S}_{2} \mathrm{Si}_{2} \mathrm{Na}$ 817.3841, found: 817.3846 .

\subsection{Synthesis of $\mathbf{9}$}

To a solution of propane-1,3-diol $8(7.60 \mathrm{~g}, 100 \mathrm{mmol})$ in $200 \mathrm{~mL}$ of anhydrous THF was added $\mathrm{NaH}$ (4.40 g, 60\% dispersion in mineral oil, $110 \mathrm{mmol}$ ) portionwise. The mixture was stirred at room temperature for $30 \mathrm{~min}$, then benzyl bromide $(18.90 \mathrm{~g}, 110 \mathrm{mmol})$ was added dropwise, then tetrabutylammonium iodide $(7.40 \mathrm{~g}, 20 \mathrm{mmol})$ was added in one portion. The mixture was heated to $60{ }^{\circ} \mathrm{C}$ and stirred overnight. After cooling, an equal volume of water was added, and the mixture was extracted with diethyl ether. The combined organic layer was washed with brine, dried over anhydrous $\mathrm{Na}_{2} \mathrm{SO}_{4}$, and filtered. The solvent was evaporated, and the resulting residue was purified by flash column chromatography on silica gel with hexane/EtOAc $=5: 1$ to afford a yellow liquid $(13.80 \mathrm{~g}, 83 \%$ yield) [45]. ${ }^{1} \mathrm{H}$ NMR (360 MHz, $\left.\mathrm{CDCl}_{3}\right) \delta(\mathrm{ppm}): 7.38-7.28(\mathrm{~m}, 5 \mathrm{H}), 4.53(\mathrm{~s}, 2 \mathrm{H}), 3.80$ (apparent q, $J=$ $7.2 \mathrm{~Hz}, 2 \mathrm{H}), 3.67(\mathrm{t}, J=7.2 \mathrm{~Hz}, 2 \mathrm{H}), 2.28(\mathrm{t}, J=3.6 \mathrm{~Hz}, 1 \mathrm{H}), 1.91-1.84(\mathrm{~m}, 2 \mathrm{H})$.

\subsection{Synthesis of $\mathbf{1 0}$}

To a solution of 3-(benzyloxy)propan-1-ol 9 (9.30 g, $56.0 \mathrm{mmol})$ in $180 \mathrm{~mL}$ of $\mathrm{CH}_{2} \mathrm{Cl}_{2}$ was added $\mathrm{PPh}_{3}(15.42 \mathrm{~g}, 58.8 \mathrm{mmol})$ and imidazole $(4.57 \mathrm{~g}, 61.6 \mathrm{mmol})$, followed by the portionwise addition of iodine $(15.63 \mathrm{~g}, 61.6 \mathrm{mmol})$ at $0{ }^{\circ} \mathrm{C}$ (ice bath). Then the reaction mixture was stirred at $\mathrm{rt}$ for 16 $h$ and then quenched with aqueous $\mathrm{Na}_{2} \mathrm{SO}_{3}$ solution. The organic phase was separated, and the aqueous phase was extracted with $\mathrm{CH}_{2} \mathrm{Cl}_{2}$. The combined organic layer was dried with $\mathrm{Na}_{2} \mathrm{SO}_{4}$ and then filtered. The solvent was evaporated, the residue was purified by flash column chromatography through silica gel eluting with hexane, obtaining a colorless liquid (12.1 g, 78\% yield) [45]. ${ }^{1} \mathrm{H}$ NMR $\left(360 \mathrm{MHz}, \mathrm{CDCl}_{3}\right) \delta(\mathrm{ppm}): 7.38-7.27(\mathrm{~m}, 5 \mathrm{H}), 4.52(\mathrm{~s}, 2 \mathrm{H}), 3.54(\mathrm{t}, J=7.2 \mathrm{~Hz}, 2 \mathrm{H}), 3.31(\mathrm{t}, J=7.2 \mathrm{~Hz}$, $2 \mathrm{H}), 2.13-2.06(\mathrm{~m}, 2 \mathrm{H})$.

\subsection{Synthesis of $\mathbf{1 1}$}

An excess of [(3-iodopropoxy)methyl]benzene 10 (10.35 g, $37.5 \mathrm{mmol})$ was added to a stirred solution of 3,5-dihydroxybenzaldehyde $6(2.07 \mathrm{~g}, 15 \mathrm{mmol})$ and cesium carbonate $(24.44 \mathrm{~g}, 75.0 \mathrm{mmol})$ in anhydrous DMF $(120 \mathrm{~mL})$. The reaction was stirred at $40{ }^{\circ} \mathrm{C}$ under argon atmosphere until 3,5-dihydroxybenzaldehyde 6 was fully consumed (TLC monitoring, $41 \mathrm{~h}$ ). Then, water was added $(60 \mathrm{~mL})$ and the crude product was extracted with EtOAc $(80 \mathrm{~mL} \times 3)$. The organic phase was washed with water ( 3 times), dried with anhydrous $\mathrm{Na}_{2} \mathrm{SO}_{3}$ and the solvent was removed under vacuum to afford the crude product as yellow liquid. The pure product was obtained by a flash column chromatography through silica gel eluting with hexane/EtOAc $=5: 1$, colorless oil $(6.07 \mathrm{~g}, 93 \%$ yield) [45]. ${ }^{1} \mathrm{H}$ NMR (360 MHz, $\left.\mathrm{CDCl}_{3}\right) \delta(\mathrm{ppm}): 9.88(\mathrm{~s}, 1 \mathrm{H}), 7.33-7.27(\mathrm{~m}, 10 \mathrm{H}), 7.00(\mathrm{~d}, J=3.6 \mathrm{~Hz}$, $2 \mathrm{H}), 6.69(\mathrm{t}, J=3.6 \mathrm{~Hz}, 1 \mathrm{H}), 4.53(\mathrm{~s}, 4 \mathrm{H}), 4.12(\mathrm{t}, J=7.2 \mathrm{~Hz}, 4 \mathrm{H}), 3.66(\mathrm{t}, J=7.2 \mathrm{~Hz}, 4 \mathrm{H}), 2.13-2.05(\mathrm{~m}$, $4 \mathrm{H}) ;{ }^{13} \mathrm{C}$ NMR $\left(91 \mathrm{MHz}, \mathrm{CDCl}_{3}\right) \delta$ (ppm): 192.1, 160.6, 151.8, 138.3, 128.4, 127.7, 127.6, 108.0, 107.7, 73.1, 66.6, 65.3, 29.6. IR (film): 3029.5, 2932.3, 1696.8, 1591.9, 1452.1, 1295.4, 1164.7, 1094.2, 734.2, $696.7 \mathrm{~cm}^{-1}$. ESI-MS m/z: 457.2 [M + Na] ${ }^{+}, 306.9,249.9,181.1,165.1,141.1,91.1 ; \mathrm{HRMS}(\mathrm{ESI}) \mathrm{m} / \mathrm{z}[\mathrm{M}+\mathrm{Na}]^{+} \mathrm{calcd}$ for $\mathrm{C}_{27} \mathrm{H}_{30} \mathrm{O}_{5} \mathrm{Na}: 457.1985$, found: 457.1984 . 


\subsection{Synthesis of $\mathbf{1 2}$}

3,5-Bis[3-(benzyloxy)propoxy]benzaldehyde $\mathbf{1 1}(3.70 \mathrm{~g}, 8.5 \mathrm{mmol})$ was dissolved in acetonitrile $(20 \mathrm{~mL})$, then a solution of $\mathrm{NaH}_{2} \mathrm{PO}_{4} \cdot 2 \mathrm{H}_{2} \mathrm{O}(358.0 \mathrm{mg}, 2.30 \mathrm{mmol})$ in water $(5.0 \mathrm{~mL})$ and $30 \% \mathrm{H}_{2} \mathrm{O}_{2}$ $(1.1 \mathrm{~mL}, 9.97 \mathrm{~mol} / \mathrm{L}, 10.97 \mathrm{mmol})$ was added. Finally, a solution of $80 \%$ purity $\mathrm{NaClO}_{2}(1.36 \mathrm{~g}, 12.0 \mathrm{mmol})$ in water $(12 \mathrm{~mL})$ was added dropwise during $2 \mathrm{~h}$ to the stirred mixture at room temperature. The $\mathrm{O}_{2}$ produced from the solution was monitored until the end of the reaction with a bubbler connected to the flask. A little amount of $\mathrm{Na}_{2} \mathrm{SO}_{3}$ was added to destroy the unreacted oxidants. The mixture was acidified with $10 \%$ aqueous $\mathrm{HCl}$, and the product was extracted using ethyl acetate $(80 \mathrm{~mL} \times 3)$. The organic phase was dried with anhydrous sodium sulfate and the solvent was removed under vacuum to afford the crude product, which was purified by a flash column chromatography through silica gel eluting with hexane/EtOAc $=1: 2$, white solid $(3.78 \mathrm{~g}, 99 \%$ yield $)[42] .{ }^{1} \mathrm{H}$ NMR $\left(360 \mathrm{MHz}, \mathrm{CDCl}_{3}\right) \delta$ (ppm): 7.32-7.24 (m, 12H), $6.67(\mathrm{br} \mathrm{s}, 1 \mathrm{H}), 4.53(\mathrm{~s}, 4 \mathrm{H}), 4.12(\mathrm{t}, J=7.2 \mathrm{~Hz}, 4 \mathrm{H}), 3.67(\mathrm{t}, J=7.2 \mathrm{~Hz}, 4 \mathrm{H})$, 2.18-2.05 (m, 4H); ${ }^{13} \mathrm{C}$ NMR (91 MHz, $\left.\mathrm{CDCl}_{3}\right) \delta(\mathrm{ppm}): 160.1,152.7,138.3,129.8,128.4,127.7,127.6$, 108.3, 107.4, 73.1, 66.6, 65.2, 29.6. IR (film): 3651.4, 3024.9, 2861.2, 1686.4, 1593.5, 1445.7, 1393.4, 1297.5, 1169.4, $1091.7 \mathrm{~cm}^{-1}$. ESI-MS m/z: 473.2 [M + Na] ${ }^{+}, 433.2,283.1,239.1,181.1,122.1 ;$ HRMS (ESI): $\mathrm{m} / \mathrm{z}$ [M $+\mathrm{Na}^{+}$calcd for $\mathrm{C}_{27} \mathrm{H}_{30} \mathrm{O}_{6} \mathrm{Na}: 473.1935$, found: 473.1937 .

\subsection{Synthesis of $\mathbf{1 3}$}

In a 10-mL round bottom flask, 3,5-bis[3-(benzyloxy)propoxy]benzoic acid 12 (5.40 g, $12.0 \mathrm{mmol}$ ), di-tert-butyl dicarbonate $(5.24 \mathrm{~g}, 24.0 \mathrm{mmol})$ and 4-dimethylaminopyridine $(439.8 \mathrm{mg}, 3.6 \mathrm{mmol})$ were dissolved in tert-butanol $(80 \mathrm{~mL})$. The reaction mixture was stirred at $40{ }^{\circ} \mathrm{C}$ under argon atmosphere until 3,5-bis[3-(benzyloxy)propoxy]benzoic acid 12 was fully consumed (TLC monitoring, $48 \mathrm{~h}$ ). Then, the solvent was removed under vacuum and the crude product was purified by flash column chromatography through silica gel eluting with hexane/AcOEt $=10: 1$, obtaining the pure product 13 as colorless oil (4.25 g, 70\% yield) [43]. ${ }^{1} \mathrm{H}$ NMR $\left(360 \mathrm{MHz}, \mathrm{CDCl}_{3}\right) \delta(\mathrm{ppm}): 7.33-7.12(\mathrm{~m}, 10 \mathrm{H}), 7.13$ $(\mathrm{d}, J=3.6 \mathrm{~Hz}, 2 \mathrm{H}), 6.60(\mathrm{t}, J=3.6 \mathrm{~Hz}, 1 \mathrm{H}), 4.52(\mathrm{~s}, 4 \mathrm{H}), 4.09(\mathrm{t}, J=7.2 \mathrm{~Hz}, 4 \mathrm{H}), 3.65(\mathrm{t}, J=7.2 \mathrm{~Hz}, 4 \mathrm{H})$, 2.10-2.04 (m, 4H), $1.58(\mathrm{~s}, 9 \mathrm{H}) ;{ }^{13} \mathrm{C} \mathrm{NMR}\left(91 \mathrm{MHz}, \mathrm{CDCl}_{3}\right) \delta(\mathrm{ppm}): 165.6,159.6,138.4,133.9,128.4$, 127.65, 127.60, 107.8, 105.8, 81.1, 73.1, 66.7, 65.1, 29.7, 28.2. IR (film): 3651.4, 2930.3, 2860.4, 1710.1, 1593.9, 1445.8, 1300.6, 1160.7, 1096.4, 731.9, $696.7 \mathrm{~cm}^{-1}$. ESI-MS m/z: $529.3[\mathrm{M}+\mathrm{Na}]^{+}, 451.3,433.2$, 283.1, 265.1, 181.1, 122.1; HRMS (ESI): $\mathrm{m} / \mathrm{z}[\mathrm{M}+\mathrm{Na}]^{+}$calcd for $\mathrm{C}_{31} \mathrm{H}_{38} \mathrm{O}_{6} \mathrm{Na}$ : 529.2561, found: 529.2555 .

\subsection{Synthesis of $\mathbf{1 4}$}

A mixture of tert-butyl 3,5-bis[3-(benzyloxy)propoxy]benzoate $13(1.40 \mathrm{~g}, 2.77 \mathrm{mmol})$ and preequilibrated $10 \% \mathrm{Pd} / \mathrm{C}(0.308 \mathrm{~g}, 0.029 \mathrm{mmol} \mathrm{Pd})$ in absolute ethanol $(20 \mathrm{~mL})$ was hydrogenated at room temperature and atmospheric pressure. When tert-butyl 3,5-bis[3-(benzyloxy)propoxy]benzoate 13 was fully consumed (TLC monitoring, $17 \mathrm{~h}$ ), the reaction mixture was filtered through celite ${ }^{\circledR}$ 545 , the filtrate was evaporated to give the crude product. The final pure product was obtained by flash column chromatography (silica gel eluting with hexane/EtOAc $=1: 2)$, white solid $(858 \mathrm{mg}, 95 \%$ yield) [46]. ${ }^{1} \mathrm{H}$ NMR $\left(360 \mathrm{MHz}, \mathrm{CDCl}_{3}\right) \delta(\mathrm{ppm}): 7.14(\mathrm{~d}, J=3.6 \mathrm{~Hz}, 2 \mathrm{H}), 6.62(\mathrm{t}, J=3.6 \mathrm{~Hz}, 1 \mathrm{H})$, 4.16-4.12 (m, 4H), $3.85(\mathrm{t}, J=7.2 \mathrm{~Hz}, 4 \mathrm{H}), 2.07-2.01(\mathrm{~m}, 4 \mathrm{H}), 1.58(\mathrm{~s}, 9 \mathrm{H}) ;{ }^{13} \mathrm{C} \mathrm{NMR}\left(91 \mathrm{MHz}, \mathrm{CDCl}_{3}\right) \delta$ (ppm): 165.4, 159.7, 134.0, 107.8, 105.8, 81.3, 65.9, 60.3, 31.9, 28.1. IR (film): 3274.5, 2967.9, 2870.6, 1705.7, 1595.1, 1447.7, 1349.7, 1299.2, 1169.9, 1058.6, 847.4, 767.4 cm-1. ESI-MS m/z: $349.2[\mathrm{M}+\mathrm{Na}]^{+}, 293.1$, 271.1, 253.1, 195.1, 122.1; HRMS (ESI): $\mathrm{m} / \mathrm{z}[\mathrm{M}+\mathrm{Na}]^{+}$calcd for $\mathrm{C}_{17} \mathrm{H}_{26} \mathrm{O}_{6} \mathrm{Na}$ : 349.1622, found: 349.1618.

\subsection{Synthesis of $\mathbf{P 2}$}

In a 100-mL Schlenk tube under nitrogen, tert-butyl 3,5-bis(3-hydroxypropoxy)benzoate 14 (1.96 g, $6.0 \mathrm{mmol}$ ) and 3-(isocyanatopropyl)triethoxysilane $15(7.42 \mathrm{~g}, 7.42 \mathrm{~mL}, 1.0 \mathrm{~g} / \mathrm{mL}, 30.0 \mathrm{mmol}$ ) were dissolved in dry THF $(20 \mathrm{~mL})$. The mixture was stirred under reflux and argon atmosphere. After $17 \mathrm{~h}$, the starting material was fully consumed (TLC monitoring). Then, the solvent was removed under 
reduced pressure and the residue was purified by flash column chromatography through silica gel eluting with hexane/EtOAc $=2: 1$ to obtain the pure product P2 as colorless oil (4.90 g, 100\% yield). ${ }^{1} \mathrm{H}$ NMR (360 MHz, $\left.\mathrm{CDCl}_{3}\right) \delta(\mathrm{ppm}): 7.12$ (broad s, 2H), $6.59(\mathrm{t}, J=3.6 \mathrm{~Hz}, 1 \mathrm{H}), 4.99(\mathrm{br} \mathrm{s}, 2 \mathrm{H}), 4.22$ $(\mathrm{t}, J=7.2 \mathrm{~Hz}, 4 \mathrm{H}), 4.05(\mathrm{t}, J=7.2 \mathrm{~Hz}, 4 \mathrm{H}), 3.81(\mathrm{q}, J=7.2 \mathrm{~Hz}, 12 \mathrm{H}), 3.18(\mathrm{q}, J=7.2 \mathrm{~Hz}, 4 \mathrm{H}), 2.09-2.04$ $(\mathrm{m}, 4 \mathrm{H}), 1.72-1.61(\mathrm{~m}, 4 \mathrm{H}), 1.57(\mathrm{~s}, 9 \mathrm{H}), 1.21(\mathrm{t}, J=7.2 \mathrm{~Hz}, 18 \mathrm{H}), 0.62(\mathrm{t}, J=7.2 \mathrm{~Hz}, 4 \mathrm{H}) ;{ }^{13} \mathrm{C} \mathrm{NMR}$ $\left(91 \mathrm{MHz} \mathrm{CDCl}_{3}\right) \delta(\mathrm{ppm}):$ 165.5, 159.7, 156.5, 133.8, 107.7, 105.8, 81.2, 64.6, 61.3, 58.4, 43.4, 28.9, 28.1, 23.3, 18.3, 7.6. IR (film): 3343.6, 2972.7, 2927.5, 1708.6, 1595.9, 15527.2, 1242.3, 1163.0, 1071.9, 953.2, $767.1 \mathrm{~cm}^{-1}$. ESI-MS m/z: $843.1[\mathrm{M}+\mathrm{Na}]^{+}, 775.4,729.3,673.3 ; \mathrm{HRMS}$ (ESI): m/z [M + Na] ${ }^{+}$calcd for $\mathrm{C}_{37} \mathrm{H}_{68} \mathrm{~N}_{2} \mathrm{O}_{14} \mathrm{Si}_{2} \mathrm{Na}$ : 843.4101, found: 843.4108.

\subsection{General Procedure for the Preparation of E-Pn 90/10 or 75/25 PMO NPs}

A solution of CTAB (250 mg, $0.686 \mathrm{mmol})$ in Mili-Q water $(120 \mathrm{~mL})$ was placed in a $250 \mathrm{~mL}$ round bottom flask, then $2 \mathrm{M} \mathrm{NaOH}(875 \mu \mathrm{L})$ was added $(1.75 \mathrm{mmol}$ of $\mathrm{NaOH})$. The mixture was stirred at $1000 \mathrm{rpm}$ at $80{ }^{\circ} \mathrm{C}$ for $50 \mathrm{~min}$. After that, the stirring speed was increased to $1400 \mathrm{rpm}$ and a mixture of 100\% E-BTSE with Pn (for 90/10: $2.00 \mathrm{mmol} E$-BTSE and 0.2 mmol Pn; for 75/25: $1.65 \mathrm{mmol} E$-BTSE and $0.55 \mathrm{mmol} \mathrm{Pn}$ ) was added rapidly under stirring. The condensation process was conducted for another $120 \mathrm{~min}$ at $80{ }^{\circ} \mathrm{C}$. Afterwards, the suspension was cooled to room temperature and the nanoparticles were collected by centrifugation (13500 rpm for $45 \mathrm{~min}$ ). The samples were then extracted with a solution of $\mathrm{NH}_{4} \mathrm{NO}_{3}(6 \mathrm{~g} / \mathrm{L}$ in $96 \% \mathrm{EtOH})$ for three times and washed successively with $96 \%$ ethanol, Mili-Q water, 96\% ethanol. The E-Pn PMO NPs were obtained as white solids [39]. E-P1 90/10 PMO NPs: ${ }^{13}$ C-CP-MAS NMR (75 MHz) $\delta$ (ppm): 165, 160, 146, 134, 107, 82, 68, 58, 33, 28, 24, 17, 13. IR $v$ (ATR) $\left(\mathrm{cm}^{-1}\right): 3311.8,2975.4,1692.3,1596.5,1449.9,1187.3,1033.1,923.2,788.5$. BET: $\mathrm{S}_{\text {BET }}=973 \mathrm{~m}^{2} \mathrm{~g}^{-1}$, $\mathrm{V}_{\text {pore }}=0.66 \mathrm{~cm}^{3} \mathrm{~g}^{-1}, \emptyset_{\text {pore }}=2.73 \mathrm{~nm}$. TGA (air, $5{ }^{\circ} \mathrm{C} / \mathrm{min}, 20-1000{ }^{\circ} \mathrm{C}$ ) residual mass $77 \%$. Zeta Potential: $\zeta=-30.9 \mathrm{mV}, \mathrm{pH}=6.80$. DLS: $1122 \mathrm{~nm}$. E-P2 90/10 PMO NPs: ${ }^{13} \mathrm{C}-\mathrm{CP}-\mathrm{MAS}$ NMR $(75 \mathrm{MHz})$ $\delta$ (ppm): 165, 160, 146, 134, 109, 81, 63, 58, 44, 28, 24, 17, 11. IR $v$ (ATR) $\left(\mathrm{cm}^{-1}\right): 3316.2,1695.5,1448.5$, 1187.9, 1035.2, 923.1, 789.2. BET: $\mathrm{S}_{\mathrm{BET}}=696 \mathrm{~m}^{2} \mathrm{~g}^{-1}, \mathrm{~V}_{\text {pore }}=0.37 \mathrm{~cm}^{3} \mathrm{~g}^{-1}, \emptyset_{\text {pore }}=2.13 \mathrm{~nm}$. TGA (air, $5{ }^{\circ} \mathrm{C} / \mathrm{min}, 20-1000{ }^{\circ} \mathrm{C}$ ) residual mass $75 \%$. Zeta Potential: $\zeta=-38.2 \mathrm{mV}, \mathrm{pH}=6.72$. DLS: $694 \mathrm{~nm}$. E-P1 75/25 PMO NPs: ${ }^{13}$ C-CP-MAS NMR (75 MHz) $\delta$ (ppm): 165, 160, 146, 134, 107, 81, 68, 58, 34, $29,18,13$. IR $v\left(\right.$ ATR) $\left(\mathrm{cm}^{-1}\right): 3359.1,1709.4,1594.2,1447.0,1035.6,924.5,790.3$. BET: $\mathrm{S}_{\mathrm{BET}}=259 \mathrm{~m}^{2} \mathrm{~g}^{-1}, \mathrm{~V}_{\text {pore }}=$ $0.17 \mathrm{~cm}^{3} \mathrm{~g}^{-1}, \emptyset_{\text {pore }}=2.59 \mathrm{~nm}$. TGA (air, $5^{\circ} \mathrm{C} / \mathrm{min}, 20-1000{ }^{\circ} \mathrm{C}$ ) residual mass $67 \%$. Zeta Potential: $\zeta=$ -25.6 mV, pH = 9.07. DLS: 742 nm. E-P2 75/25 PMO NPs: ${ }^{13}$ C-CP-MAS NMR (75 MHz) $\delta(p p m): ~ 166$, 160, 158, 146, 134, 111, 105, 81, 64, 58, 44, 28, 24, 18, 11. IR $v$ (ATR) $\left(\mathrm{cm}^{-1}\right): 3340.1,1692.2,1447.3,1188.5$, 1035.8, 922.5, 791.1. BET: $\mathrm{S}_{\mathrm{BET}}=329 \mathrm{~m}^{2} \mathrm{~g}^{-1}, \mathrm{~V}_{\text {pore }}=0.26 \mathrm{~cm}^{3} \mathrm{~g}^{-1}, \emptyset_{\text {pore }}=2.74 \mathrm{~nm}$. TGA $\left(\right.$ air, $5^{\circ} \mathrm{C} / \mathrm{min}$, 20-1000 ${ }^{\circ} \mathrm{C}$ ) residual mass 63\%. Zeta Potential: $\zeta=-42.3 \mathrm{mV}, \mathrm{pH}=6.85$. DLS: $562 \mathrm{~nm}$.

\section{Results and Discussion}

\subsection{Synthesis of the Disilylated Precursors $\mathbf{P 1}$ and $\mathbf{P 2}$}

The synthesis of $\mathbf{P 1}$ is depicted in Scheme 1. We first converted commercial 3-buten-1-ol $\mathbf{1}$ into but-3-en-1-yl methanesulfonate 2 [40]. The dialkylation of commercial 3,5-dihydroxybenzaldehyde, 3 , with the mesylate 2 , in the presence of cesium carbonate in DMF at $40{ }^{\circ} \mathrm{C}$, afforded 4 in $78 \%$ isolated yield after chromatographic purification [41]. The aldehyde 4 was oxidized to the carboxylic acid 5 by using $\mathrm{NaClO}_{2}, 30 \% \mathrm{H}_{2} \mathrm{O}_{2}$ and $\mathrm{NaH}_{2} \mathrm{PO}_{4}$ in aqueous acetonitrile at room temperature [42]. Subsequent treatment of 5 with $\mathrm{Boc}_{2} \mathrm{O}$ and 4-dimethylaminopyridine (DMAP) in tert-butanol at room temperature provided the ester 6 in 85\% isolated yield [43]. Finally, the precursor P1 was obtained in 70\% isolated yield by the versatile thiol-alkene click reaction between dialkene 6 and (3-mercaptopropyl)triethoxysilane 7 under irradiation at $365 \mathrm{~nm}$ using 2,2-dimethoxy1,2-diphenylethanone (DMPA) as initiator [44]. 


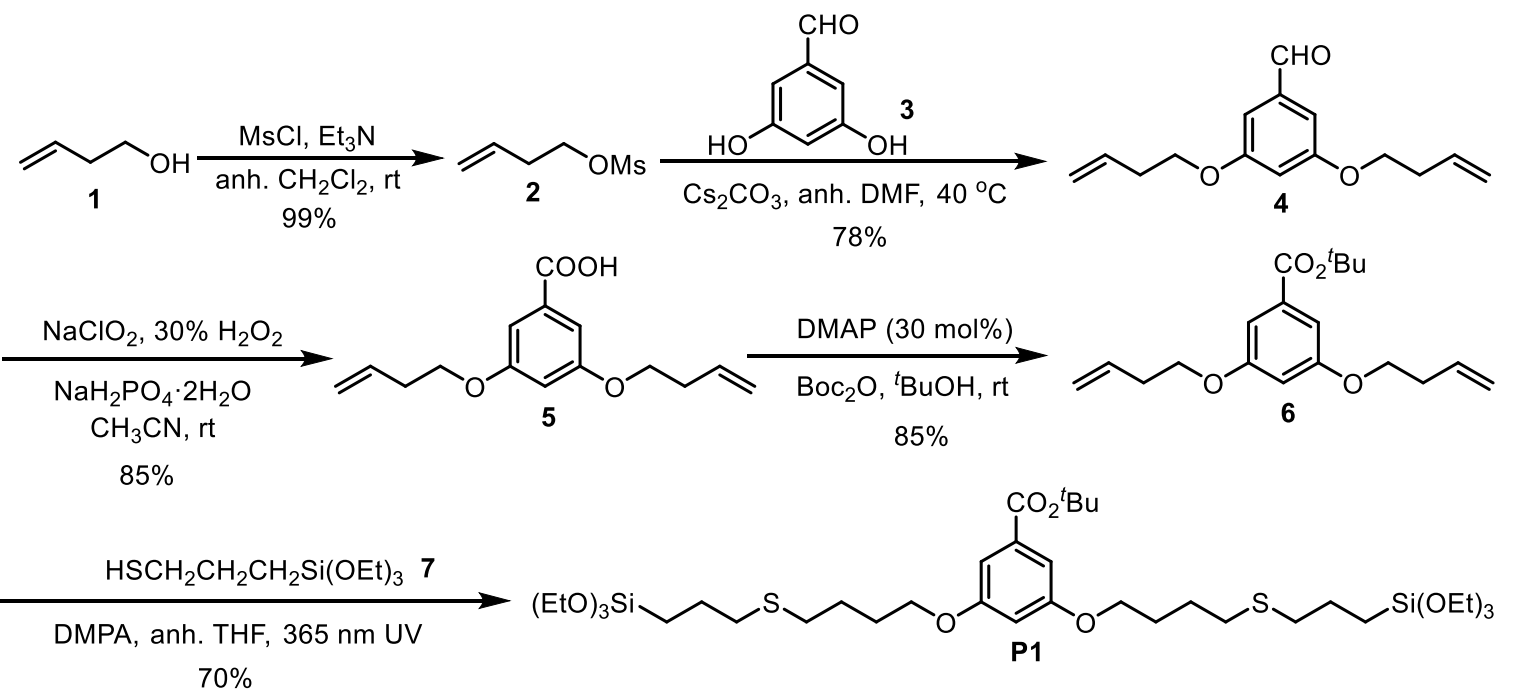

Scheme 1. Synthesis of precursor P1.

In Scheme 2, we summarize the preparation of precursor P2. We first synthesized [(3iodopropoxy)methyl)]benzene 10 (65\% overall yield) from the commercially available 1,3-propanediol 8 through the monobenzylated intermediate 9 [45]. Then, commercial 3,5-dihydroxybenzaldehyde 3 was dialkylated with the iodide $\mathbf{1 0}$ to obtain the aldehyde $\mathbf{1 1}$. The next steps were oxidation to the carboxylic acid 12, formation of tert-butyl ester 13, followed by deprotection of benzylic ethers, leading to the obtention of diol $\mathbf{1 4}$ in high yield [46]. Subsequent treatment of this compound with the silylated isocyanate $\mathbf{1 5}$ provided the disilylated carbamate precursor $\mathbf{P} 2$ in quantitative yield.
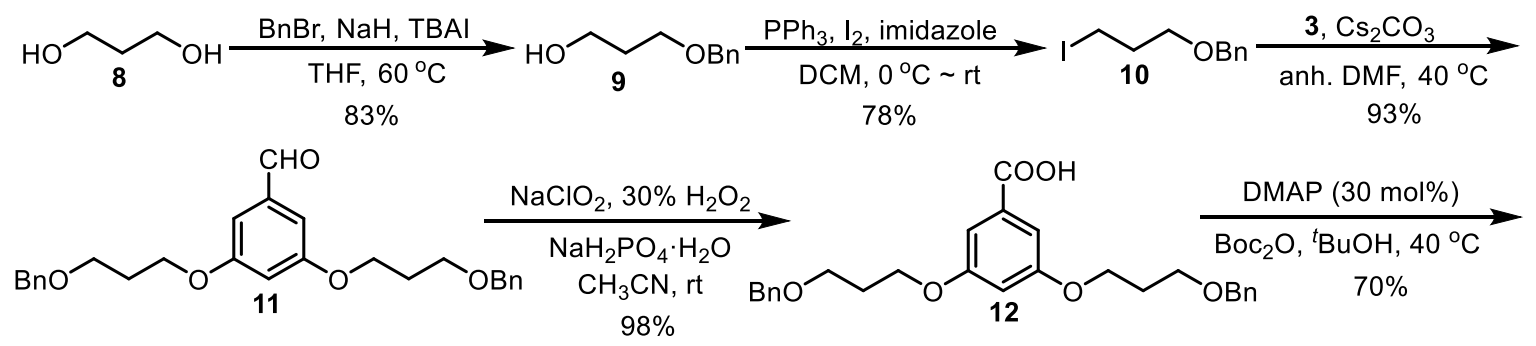

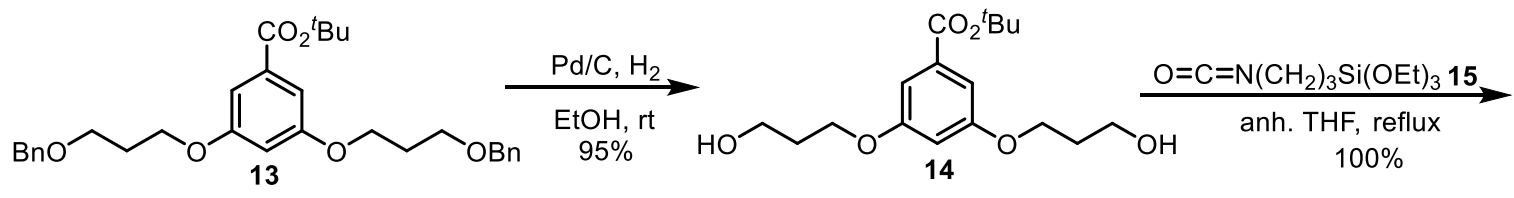<smiles>CCO[SiH2]CCCNC(=O)OCCCOc1cc(OCCCOC(=O)NCCC[SiH2]CC)cc(C(=O)OCC)c1</smiles>

Scheme 2. Synthesis of precursor P2.

\subsection{Preparation and Characterization of E-Pn 90/10 PMO NPs and E-Pn 75/25 PMO NPs}

With those precursors P1 and P2 in hand, a series of E-Pn PMO NPs were prepared by mixing the corresponding disilylated precursor and (E)-BTSE (named E) as major reagent [38] The syntheses were performed in Mili-Q water with CTAB as template under basic catalysis $(\mathrm{NaOH})$. The micellar solution was prepared at $80{ }^{\circ} \mathrm{C}$ for $50 \mathrm{~min}$ with a stirring speed adjusted at $1000 \mathrm{rpm}$. Then, the stirring speed was enhanced to $1400 \mathrm{rpm}$, a mixture of (E)-BTSE (or E) and different amounts of the corresponding disilylated precursor Pn was quickly added and the mixture was left to react for $120 \mathrm{~min}$ at $80{ }^{\circ} \mathrm{C}$ [39]. 
E-Pn PMO NPs were collected by centrifugation. The template was then removed by washing with a solution of $\mathrm{NH}_{4} \mathrm{NO}_{3}(6 \mathrm{~g} / \mathrm{L}$ in $96 \% \mathrm{EtOH})$. Herein, two different ratios of $\mathbf{E} / \mathbf{P n}$ were prepared, namely $90 / 10$ and $75 / 25$, in order to investigate the influence on the size, morphology and textural properties of the resulting nanomaterials (Scheme 3 ).<smiles>CCO[Si]/C=C/[SiH](OCC)OCC</smiles>

$100-x \%$

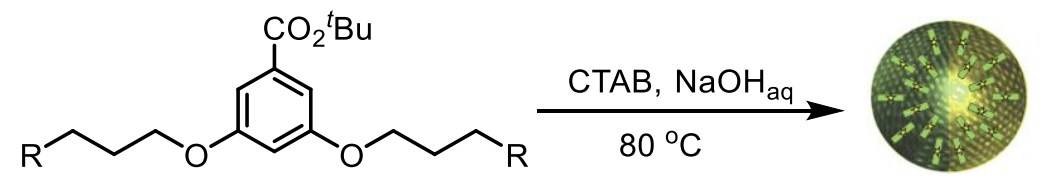

Pn

Mixed PMO NPs

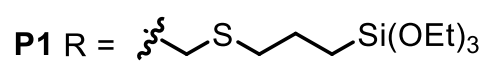

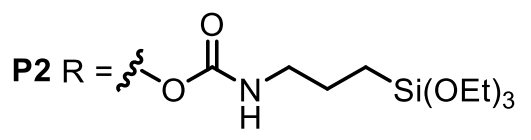

Scheme 3. Preparation of E-Pn PMO NPs.

All the mixed PMO NPs were characterized by transmission electron microscopy (TEM), nitrogen-sorption measurements (BET), dynamic light scattering (DLS), zeta-potential, thermogravimetric analysis (TGA), ${ }^{13} \mathrm{C} C \mathrm{CP}$ MAS solid state NMR, Fourier-transform infrared spectroscopy (FTIR) and small-angle X-ray diffraction ( $p$-XRD). Table 1 shows the physical data of these new materials.

Table 1. Some data of E-Pn PMO NPs.

\begin{tabular}{|c|c|c|c|c|c|c|c|}
\hline \multirow[b]{2}{*}{ Material } & \multicolumn{3}{|c|}{$\mathrm{N}_{2}$-Sorption Measurements } & \multirow{2}{*}{$\begin{array}{c}\text { Particles } \\
\text { Size }(\mathbf{n m})^{c}\end{array}$} & \multicolumn{2}{|c|}{ Zeta Potential } & \multirow[b]{2}{*}{ TGA $^{d}$} \\
\hline & $\begin{array}{c}S_{\mathrm{BET}} \\
\left(\mathrm{m}^{2} \mathrm{~g}^{-1}\right)\end{array}$ & $\begin{array}{c}V_{\text {pore }} \\
\left(\mathrm{cm}^{3} \mathrm{~g}^{-1}\right)^{a}\end{array}$ & $\begin{array}{l}\emptyset_{\text {pore }} \\
(\mathrm{nm})^{b}\end{array}$ & & $\mathrm{pH}$ & $(\mathrm{mV})$ & \\
\hline E-P190/10 PMO NPs & 973 & 0.66 & 2.7 & 1122 & 6.80 & -30.9 & $77 \%$ \\
\hline E-P290/10 PMO NPs & 696 & 0.37 & 2.1 & 694 & 6.72 & -38.2 & $75 \%$ \\
\hline E-P175/25 PMO NPs & 259 & 0.17 & 2.6 & 742 & 9.07 & -25.6 & $67 \%$ \\
\hline E-P275/25 PMO NPs & 329 & 0.23 & 2.7 & 562 & 6.85 & -42.3 & $63 \%$ \\
\hline
\end{tabular}

${ }^{a}$ Determined by BET at saturation of $\mathrm{p} / \mathrm{p}^{\circ}=0.98 .{ }^{b}$ Pore diameter determined with $\mathrm{BJH} .{ }^{c}$ Hydrodynamic diameters, DLS. ${ }^{d}$ Residual mass by TGA (heating rate $10^{\circ} \mathrm{C} / \mathrm{min}, 20$ to $1000^{\circ} \mathrm{C}$ ).

The morphologies of E-Pn PMO NPs were first investigated by TEM (Figure 2). All the nanomaterials were obtained as rodlike-shape nanoparticles, similarly to the parent pure E PMO NPs [11], with the length of nanorods ranging from 400 to $600 \mathrm{~nm}$. The hydrodynamic diameters were determined by DLS and the obtained values (from 560 to $1100 \mathrm{~nm}$, Table 1) are consistent with the sizes found by TEM. The zeta potentials (Table 1 ) were determined in water and gave negative values due to the presence of deprotonated silanol groups at the surface of the nanorods. The high values are indicative of stability. 


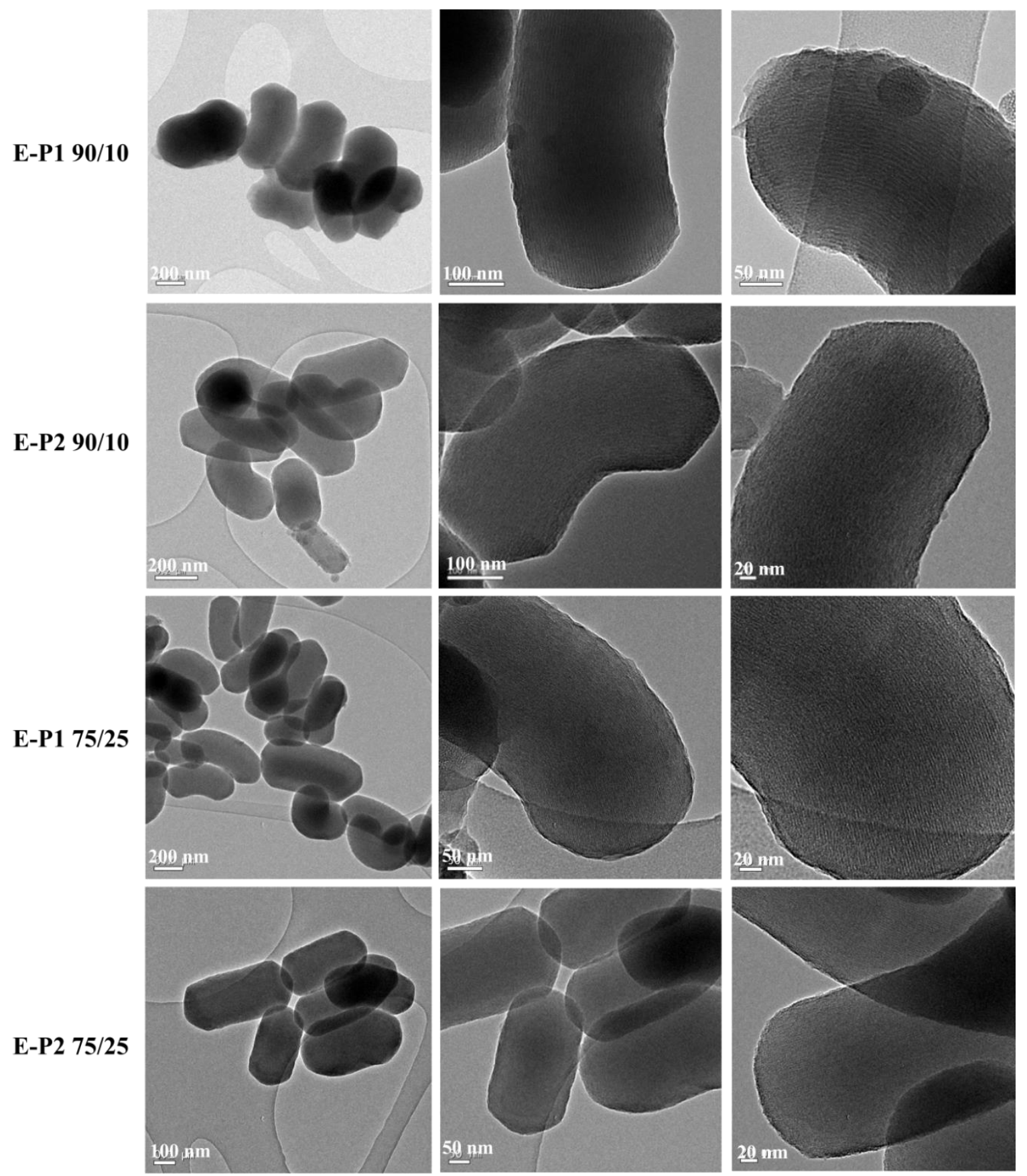

Figure 2. TEM images of E-Pn PMO NPs.

The porosity of the nanomaterials was proved by $\mathrm{N}_{2}$-sorption experiments (Figure 3). Note that whereas the $S_{\mathrm{BET}}$ for materials formed with $25 \%$ of Pn were found between 259 and $329 \mathrm{~m}^{2} \mathrm{~g}^{-1}$, E-Pn 90/10 PMO NPs presented high specific surface areas ranging from 696 to $973 \mathrm{~m}^{2} \mathrm{~g}^{-1}$ (Table 1), comparable to or even higher than pure E PMO nanorods (already synthesized) [11] which showed a specific surface area of $800 \mathrm{~m}^{2} \mathrm{~g}^{-1}$. E-P1 90/10 PMO NPs presented a type IV isotherm with a small hysteresis in agreement with a mesoporous structure of high specific surface area and pore size of 2.7 nm. E-P2 90/10 PMO NPs showed an isotherm between type I and IV. The microporosity is present with a pore size of $2.1 \mathrm{~nm}$ and a lower specific surface area than E-P1 90/10 PMO NPs of $696 \mathrm{~m}^{2} \mathrm{~g}^{-1}$. 

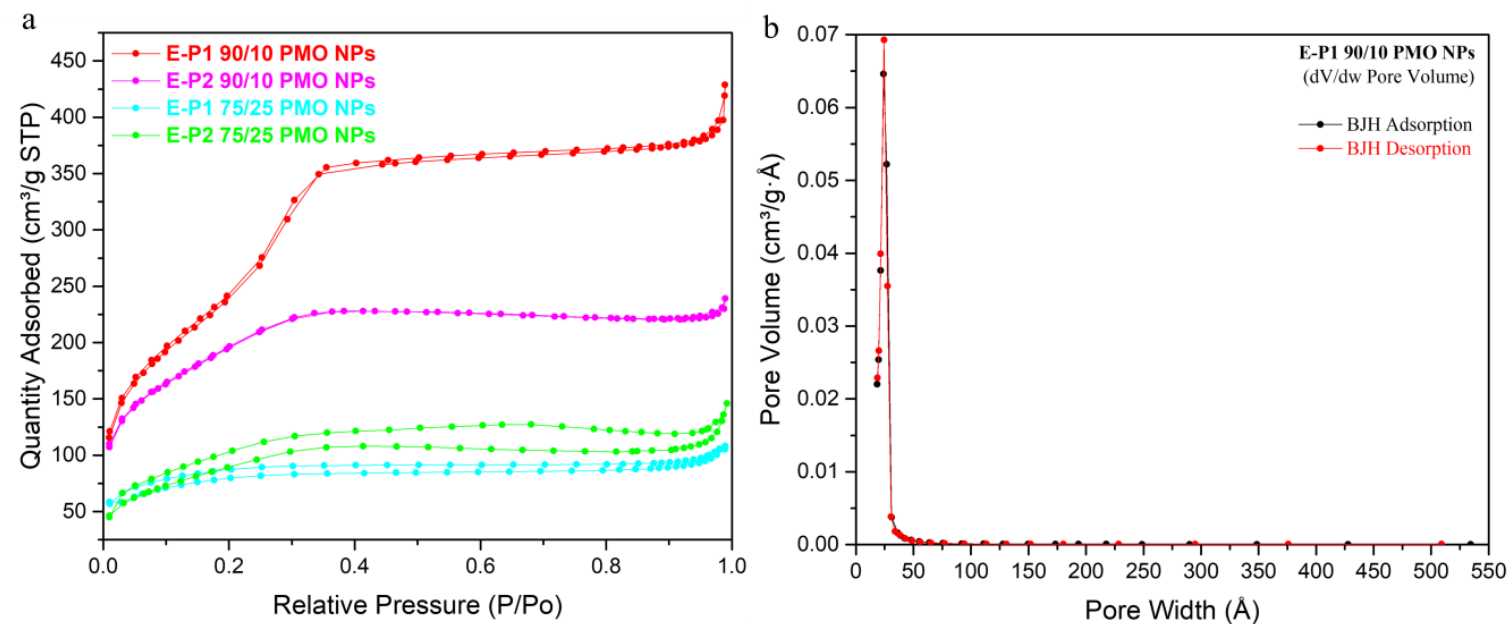

Figure 3. (a) $\mathrm{N}_{2}$-sorption isotherms of E-Pn PMO NPs; (b) BJH dV/dw Pore Volume of E-P1 90/10 PMO NPs.

In the TGA analyses (Figure 4), the loss of mass corresponding to adsorbed water (up to $120^{\circ} \mathrm{C}$ ) was observed first. The loss of the tert-butoxycarbonyl group took place from $150{ }^{\circ} \mathrm{C}$ to $300{ }^{\circ} \mathrm{C}$. Thus, this group was found to be very stable in the materials in solid dry state. Then, at higher temperatures, the decomposition of the remaining organic part of the materials occurred.

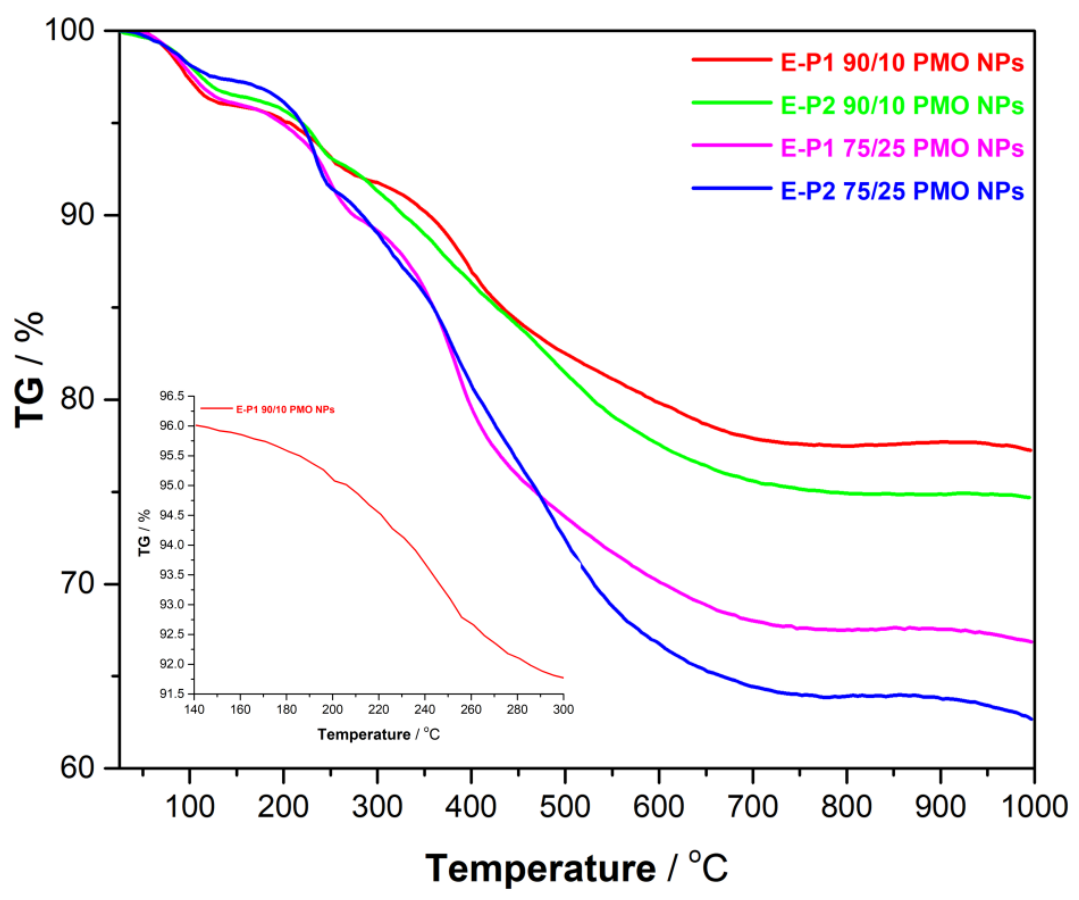

Figure 4. TGA of E-Pn PMO NPs.

${ }^{13} \mathrm{C}$ CP MAS solid-state NMR of these new nanomaterials were also recorded. The superposition of the ${ }^{13}$ C NMR spectra of Pn in solution and of E-Pn PMO NPs in solid state showed a good match, suggesting thereby that the integrity of the organic moiety of the precursor Pn had been preserved (Figure 5). For E-P1 PMO NPs, the characteristic signals (in ppm) appear at about 165 (C=O of ester), $81(\mathrm{Cq}$ of $t \mathrm{Bu}), 29\left(\mathrm{CH}_{3}\right.$ of $\left.t \mathrm{Bu}\right), 13\left(\mathrm{CH}_{2}-\mathrm{Si}\right)$, together with the carbons of the aromatic ring and the methylenic carbons of the linker. A similar pattern is observed for E-P2 PMO NPs, with additional signal at about 158 ( $\mathrm{C}=\mathrm{O}$ of carbamate). Moreover, the intense signal at about $146 \mathrm{ppm}$ in both spectra is due to the olefinic carbons of $E$-BTSE. 


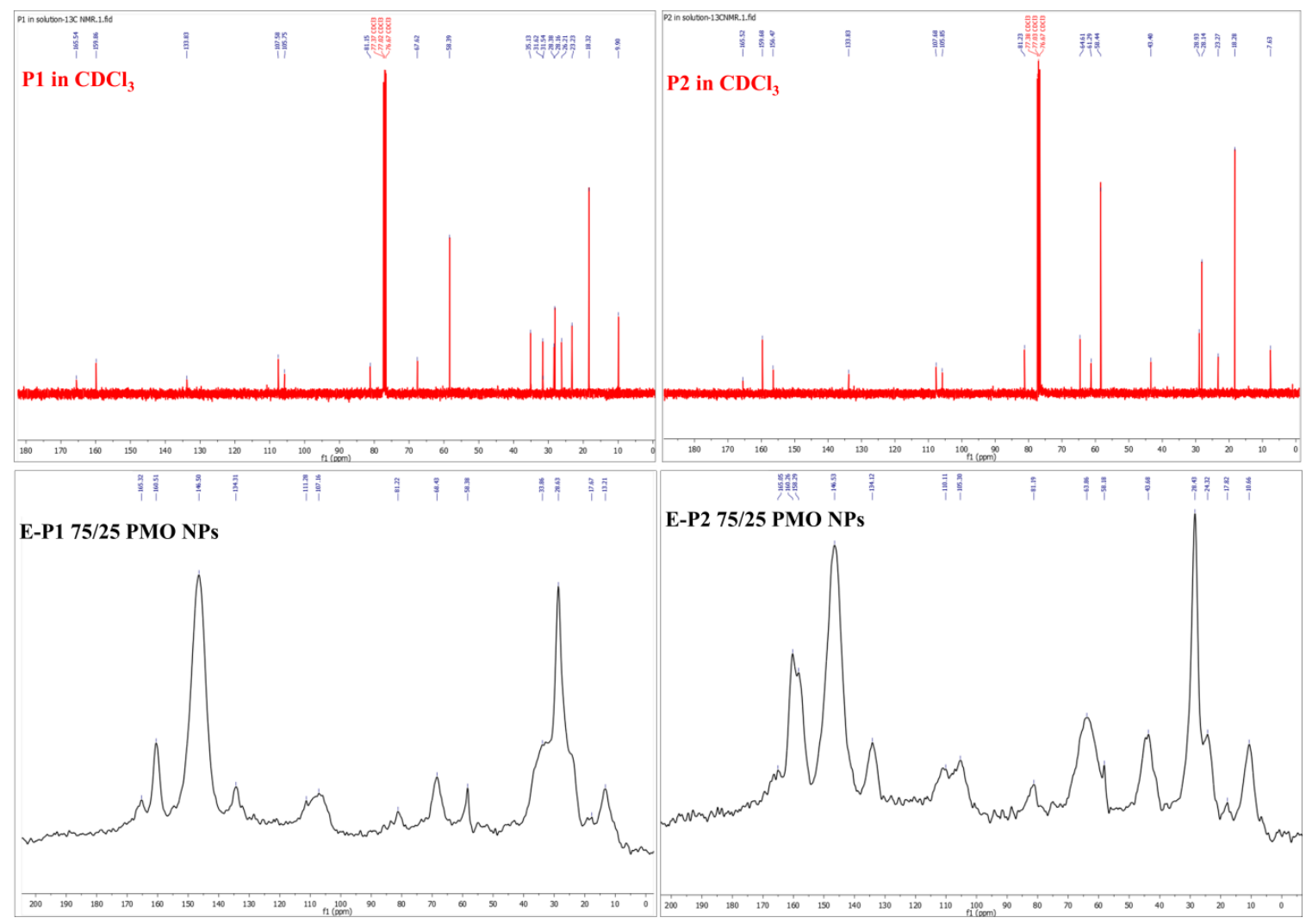

Figure 5. ${ }^{13} \mathrm{C}$ NMR of Pn in $\mathrm{CDCl}_{3}$ (above) and ${ }^{13} \mathrm{C} C P$ MAS NMR of E-Pn 75/25 PMO NPs (below).

FTIR spectra of precursors P1 and P2 (Figure 6) show the presence of tert-butyl group (2974/2973 $\left.\mathrm{cm}^{-1}, v \mathrm{CH}_{3}\right)$, carbonyl group $\left(1713 \mathrm{~cm}^{-1}, v \mathrm{C}=\mathrm{O}\right.$ of ester in $\mathbf{P 1}$; broad signal at $1709 \mathrm{~cm}^{-1}$ in $\mathbf{P 2}$ for overlapped $\mathrm{C}=\mathrm{O}$ from the ester and carbamate) and silicon-oxygen bond ( $v \mathrm{Si}-\mathrm{O}$ at $1076 / 1072$ $\mathrm{cm}^{-1}$ ). The corresponding signals are also visible in E-Pn 75/25 PMO NPs (Figure 6), with a shift for the carbonyl group to 1709/1693 $\mathrm{cm}^{-1}$. The absorption of $\mathrm{C}=\mathrm{O}$ in the case of E-P1 90/10 PMO NPs is barely visible due to the high dilution of the precursor. The intense and broad band at $1200-1000 \mathrm{~cm}^{-1}$ $(v \mathrm{Si}-\mathrm{O}-\mathrm{Si}$ ) is characteristic of a well condensed material.
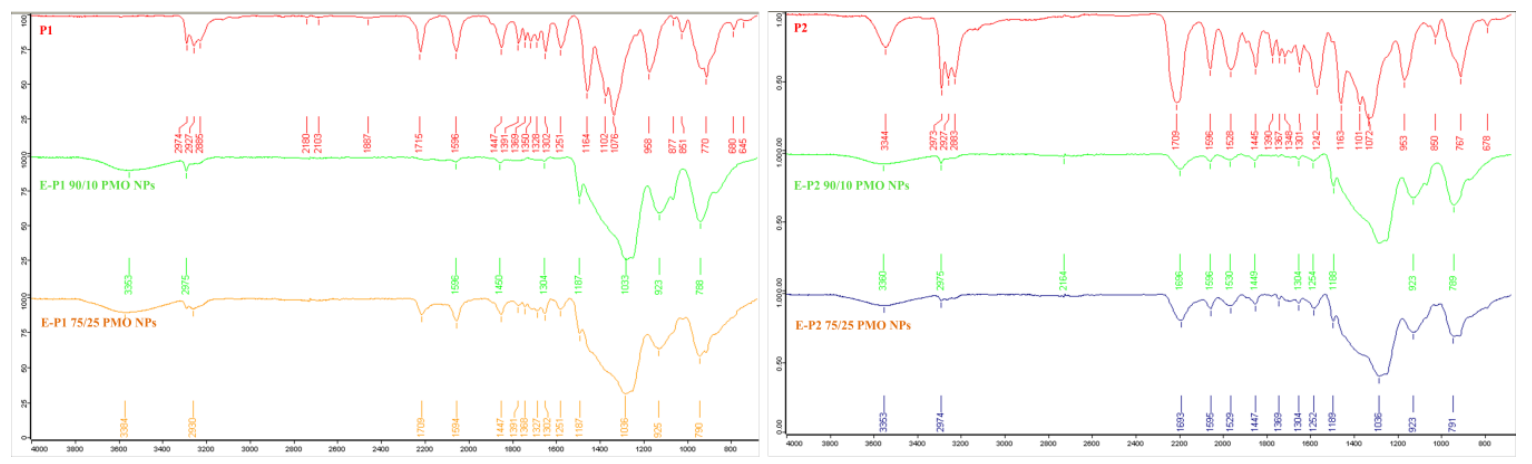

Figure 6. FTIR of Pn and E-Pn PMO NPs.

The amall angle X-ray diffraction ( $p$-XRD) analyses show an organized porosity for E-P1 90/10 PMO NPs typical for a hexagonal 2D symmetry with a sharp Bragg peak $[(1,0,0)]$ and the two first harmonics $[(1,1,0)$ and $(2,0,0)]$. The other mixed E-Pn PMO NPs are disordered, with a broad non-structured band typical of worm-like mesoporosity (Figure 7). 


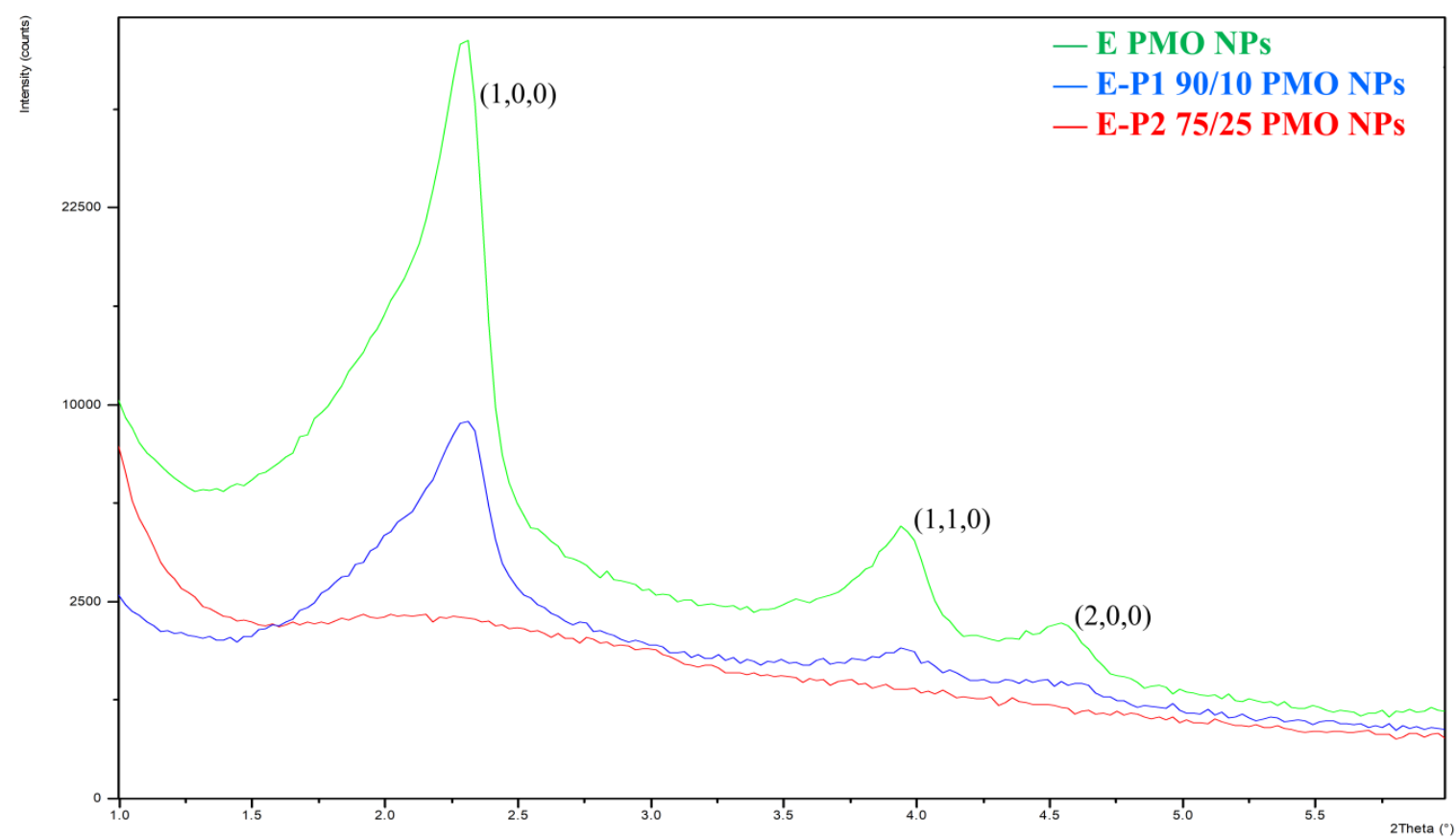

Figure 7. Small angle XRD diagram of E PMO NPs (green line), E-P1 90/10 (blue line), E-P1 75/25 (red line).

As the synthesis of PMO NPs with large and flexible organic bridging groups within the mesoporous wall is still a challenge, the development of these new mixed periodic mesoporous organosilica nanoparticles is growing. The subsequent hydrolysis of the tert-butyl ester groups in those materials would give rise to mixed PMO NPs bearing acidic and coordinating carboxyl groups, which are very relevant for potential applications in nanomedicine for $\mathrm{pH}$-triggered drug delivery $[47,48]$ or in catalysis [49].

\section{Conclusions}

In summary, novel mixed E-Pn PMO NPs have been prepared by sol-gel co-condensation of E-1,2-bis(triethoxysilyl)ethylene ((E)-BTSE or E) with previously synthesized disilylated tert-butyl 3,5-dialkoxybenzoates bearing either sulfide (precursor P1) or carbamate (precursor P2) functionalities in the linker. Two different ratios of E/Pn were examined, namely $90 / 10$ and 75/25, in order to investigate the influence on the size, morphology and textural properties of the resulting nanomaterials. The syntheses were performed in Mili-Q water with CTAB as micellar template under basic catalysis $(\mathrm{NaOH})$ at $80^{\circ} \mathrm{C}$ under magnetic stirring.

The mixed E-Pn PMO NPs were characterized by TEM, DLS, zeta-potential, nitrogen-sorption measurements (BET), TGA, FTIR, ${ }^{13} \mathrm{C} \mathrm{CP}$ MAS solid state NMR and $p$-XRD. All the nanomaterials were obtained as mesoporous rodlike-shape nanoparticles, similarly to the parent pure E PMO NPs, the length of nanorods ranging from $400-600 \mathrm{~nm}$ as demonstrated by TEM. The integrity of the organic skeleton of the corresponding precursor Pn has been preserved in the nanomaterials as shown by FTIR and ${ }^{13} \mathrm{C}$ CP MAS solid state NMR. Remarkably, whereas the specific surface for E-Pn 75/25 PMO NPs were found between 260 and $330 \mathrm{~m}^{2} \mathrm{~g}^{-1}$, E-Pn 90/10 PMO NPs presented high specific surface areas ranging from 700 to $970 \mathrm{~m}^{2} \mathrm{~g}^{-1}$, comparable or even higher than pure E PMO nanorods. Moreover, XRD analyses showed an organized porosity for E-P1 90/10 PMO NPs typical of hexagonal 2D symmetry. The other materials were disordered with a broad non-structured band that indicated worm-like mesoporosity. 
Mixed PMO NPs bearing acidic and coordinating carboxyl groups resulting from the ester hydrolysis would be very relevant for potential applications in catalysis or in nanomedicine for $\mathrm{pH}$-triggered drug delivery.

Author Contributions: Conceptualization, J.-O.D. and R.P.; methodology, H.L.; formal analysis, H.L., R.P. and J.-O.D.; writing - original draft preparation, H.L., J.-O.D. and R.P.; writing-review and editing, J.-O.D., L.R., C.C. and R.P.; supervision, J.-O.D., and R.P. All authors have read and agreed to the published version of the manuscript.

Funding: This research was funded by China Scholarship Council (CSC) (H.L., No. 201606890025), Ministerio de Ciencia, Innovación y Universidades (MCIU) of Spain (Project RTI2018-097853-B-I00 and RED2018-102387-T), Ministerio de Economía, Industria y Competitividad (MINECO) of Spain (Projects CTQ2014-53662-P and CTQ2016-81797-REDC), DURSI-Generalitat de Catalunya (Project SGR2017-0465) and CNRS (France).

Acknowledgments: The authors are grateful to China Scholarship Council (CSC) for predoctoral scholarship to H.L.

Conflicts of Interest: The authors declare no conflict of interest.

\section{References}

1. Hoffmann, F.; Cornelius, M.; Morell, J.; Fröba, M. Silica-Based Mesoporous Organic-Inorganic Hybrid Materials. Angew. Chem. Int. Ed. 2006, 45, 3216-3251. [CrossRef]

2. Hunks, W.J.; Ozin, G.A. Challenges and Advances in the Chemistry of Periodic Mesoporous Organosilicas (PMOs). J. Mater. Chem. 2005, 15, 3716-3724. [CrossRef]

3. Shea, K.J.; Loy, D.A. Bridged Polysilsesquioxanes. Molecular-Engineered Hybrid Organic-Inorganic Materials. Chem. Mater. 2001, 13, 3306-3319. [CrossRef]

4. Manchanda, A.S.; Kruk, M. Synthesis of Large-pore Face-centered-cubic Periodic Mesoporous Organosilicas with Unsaturated Bridging Groups. Microporous Mesoporous Mater. 2016, 222, 153-159. [CrossRef]

5. Park, S.S.; Moorthy, M.S.; Ha, C.S. Periodic Mesoporous Organosilicas for Advanced Applications. NPG Asia Mater. 2014, 6. [CrossRef]

6. Mizoshita, N.; Tani, T.; Inagaki, S. Syntheses, Properties and Applications of Periodic Mesoporous Organosilicas Prepared from Bridged Organosilane Precursors. Chem. Soc. Rev. 2011, 40, 789-800. [CrossRef]

7. Qian, K.; Liu, F.; Yang, J.; Huang, X.; Gu, W.; Jambhrunkar, S.; Yuan, P.; Yu, C. Pore Size-optimized Periodic Mesoporous Organosilicas for the Enrichment of Peptides and Polymers. RSC Adv. 2013, 3, 14466-14472. [CrossRef]

8. Chen, Y.; Meng, Q.; Wu, M.; Wang, S.; Xu, P.; Chen, H.; Li, Y.; Zhang, L.; Wang, L.; Shi, J. Hollow Mesoporous Organosilica Nanoparticles: A Generic Intelligent Framework-Hybridization Approach for Biomedicine. J. Am. Chem. Soc. 2014, 136, 16326-16334. [CrossRef]

9. Croissant, J.G.; Cattoën, X.; Durand, J.-O.; Wong Chi Man, M.; Khashab, N.M. Organosilica Hybrid Nanomaterials with a High Organic Content: Syntheses and Applications of Silsesquioxanes. Nanoscale 2016, 8, 19945-19972. [CrossRef]

10. De Canck, E.; Ascoop, I.; Sayari, A.; Van Der Voort, P. Periodic Mesoporous Organosilicas Functionalized with a Wide Variety of Amines for $\mathrm{CO}_{2}$ Adsorption. Phys. Chem. Chem. Phys. 2013, 15, 9792-9799. [CrossRef]

11. Croissant, J.G.; Cattoën, X.; Wong Chi Man, M.; Gallud, A.; Raehm, L.; Trens, P.; Maynadier, M.; Durand, J.-O. Biodegradable Ethylene-Bis(Propyl)Disulfide-Based Periodic Mesoporous Organosilica Nanorods and Nanospheres for Efficient in-vitro Drug Delivery. Adv. Mater. 2014, 26, 6174-6180. [CrossRef] [PubMed]

12. Noureddine, A.; Trens, P.; Toquer, G.; Cattoën, X.; Wong Chi Man, M. Tailoring the Hydrophilic/Lipophilic Balance of Clickable Mesoporous Organosilicas by the Copper-Catalyzed Azide-Alkyne Cycloaddition Click-Functionalization. Langmuir 2014, 30, 12297-12305. [CrossRef]

13. Corma, A.; Díaz, U.; Arrica, M.; Fernández, E.; Ortega, Í. Organic-Inorganic Nanospheres with Responsive Molecular Gates for Drug Storage and Release. Angew. Chem. Int. Ed. 2009, 48, 6247-6250. [CrossRef] [PubMed]

14. Du, X.; Kleitz, F.; Li, X.; Huang, H.; Zhang, X.; Qiao, S.Z. Disulfide-Bridged Organosilica Frameworks: Designed, Synthesis, Redox-Triggered Biodegradation, and Nanobiomedical Applications. Adv. Funct. Mater. 2018, 28, 1707325. [CrossRef] 
15. Omar, H.; Moosa, B.; Alamoudi, K.; Anjum, D.H.; Emwas, A.H.; El Tall, O.; Vu, B.; Tamanoi, F.; AlMalik, A.; Khashab, N.M. Impact of Pore-Walls Ligand Assembly on the Biodegradation of Mesoporous Organosilica Nanoparticles for Controlled Drug Delivery. ACS Omega 2018, 3, 5195-5201. [CrossRef] [PubMed]

16. Bürglová, K.; Noureddine, A.; Hodačová, J.; Toquer, G.; Cattoën, X.; Wong Chi Man, M. A General Method for Preparing Bridged Organosilanes with Pendant Functional Groups and Functional Mesoporous Organosilicas. Chem. Eur. J. 2014, 20, 10371-10382. [CrossRef]

17. Gomes, A.C.; Lourenço, M.A.; Bruno, S.M.; Ferreira, P.; Valente, A.A.; Pillinger, M.; Gonçalves, I.S. Post-synthetic Modification of Crystal-like Periodic Mesoporous Phenylene-silica with Ferrocenyl Groups. J. Organomet. Chem. 2014, 751, 501-507. [CrossRef]

18. Urata, C.; Yamada, H.; Wakabayashi, R.; Aoyama, Y.; Hirosawa, S.; Arai, S.; Takeoka, S.; Yamauchi, Y.; Kuroda, K. Aqueous Colloidal Mesoporous Nanoparticles with Ethenylene-Bridged Silsesquioxane Frameworks. J. Am. Chem. Soc. 2011, 133, 8102-8105. [CrossRef]

19. Doustkhah, E.; Mohtasham, H.; Farajzadeh, M.; Rostamnia, S.; Wang, Y.; Arandiyan, H.; Assadi, M.H.N. Organosiloxane Tunability in Mesoporous Organosilica and Punctuated Pd Nanoparticles Growth; Theory and Experiment. Microporous Mesoporous Mater. 2020, 293, 109832. [CrossRef]

20. Du, X.; Li, X.; Xiong, L.; Zhang, X.; Kleitz, F.; Qiao, S.Z. Mesoporous Silica Nanoparticles with Organo-bridged Silsesquioxane Framework as Innovative Platforms for Bioimaging and Therapeutic Agent Delivery. Biomaterials 2016, 91, 90-127. [CrossRef]

21. Croissant, J.G.; Fatieiev, Y.; Almalik, A.; Khashab, N.M. Mesoporous Silica and Organosilica Nanoparticles: Physical Chemistry, Biosafety, Delivery Strategies, and Biomedical Applications. Adv. Healthc. Mater. 2018, 7, 1700831. [CrossRef] [PubMed]

22. Kumar, P.; Guliants, V.V. Periodic Mesoporous Organic-inorganic Hybrid Materials: Applications in Membrane Separations and Adsorption. Microporous Mesoporous Mater. 2010, 132, 1-14. [CrossRef]

23. Croissant, J.G.; Cattoën, X.; Man, M.W.C.; Durand, J.-O.; Khashab, N.M. Syntheses and Applications of Periodic Mesoporous Organosilica Nanoparticles. Nanoscale 2015, 7, 20318-20334. [CrossRef] [PubMed]

24. Djojoputro, H.; Zhou, X.F.; Qiao, S.Z.; Wang, L.Z.; Yu, C.Z.; Lu, G.Q. Periodic Mesoporous Organosilica Hollow Spheres with Tunable Wall Thickness. J. Am. Chem. Soc. 2006, 128, 6320-6321. [CrossRef] [PubMed]

25. Cho, E.-B.; Kim, D.; Jaroniec, M. Preparation of Mesoporous Benzene-silica Nanoparticles. Microporous Mesoporous Mater. 2009, 120, 252-256. [CrossRef]

26. Liu, J.; Bai, S.; Zhong, H.; Li, C.; Yang, Q. Tunable Assembly of Organosilica Hollow Nanospheres. J. Phys. Chem. C 2010, 114, 953-961. [CrossRef]

27. Guan, B.; Cui, Y.; Ren, Z.; Qiao, Z.; Wang, L.; Liu, Y.; Huo, Q. Highly Ordered Periodic Mesoporous Organosilica Nanoparticles with Controllable Pore Structures. Nanoscale 2012, 4, 6588-6596. [CrossRef]

28. Qiao, S.Z.; Yu, C.Z.; Xing, W.; Hu, Q.H.; Djojoputro, H.; Lu, G.Q. Synthesis and Bio-adsorptive Properties of Large-pore Periodic Mesoporous Organosilica Rods. J. Am. Chem. Soc. 2005, 17, 6172-6176. [CrossRef]

29. Cho, E.-B.; Kim, D.; Jaroniec, M. Monodisperse Particles of Bifunctional Periodic Mesoporous Organosilica. J. Phys. Chem. C 2008, 112, 4897-4902. [CrossRef]

30. Mohanty, P.; Landskron, K. Periodic Mesoporous Organosilica Nanorice. Nanoscale Res. Lett. 2009, 4, $169-172$. [CrossRef]

31. Mohanty, P.; Landskron, K. Simple Systematic Synthesis of Periodic Mesoporous Organosilica Nanoparticles with Adjustable Aspect Ratios. Nanoscale Res. Lett. 2009, 4, 1524-1529. [CrossRef] [PubMed]

32. Zhang, M.; Li, Y.; Bi, L.; Zhuang, W.; Wang, S.; Chen, Y.; Li, B.; Yang, Y. Preparation of Helical Mesoporous Ethenylene-silica Nanofibers with Lamellar Mesopores on Their Surface. Chin. J. Chem. 2011, 29, 933-941. [CrossRef]

33. Fatieiev, Y.; Croissant, J.G.; Alamoudi, K.; Khashab, N.M. Cellular Internalization and Biocompatibility of Periodic Mesoporous Organosilica Nanoparticles with Tunable Morphologies: From Nanospheres to Nanowires. ChemPlusChem 2017, 82, 631-637. [CrossRef] [PubMed]

34. Croissant, J.G.; Cattoën, X.; Man, M.W.C.; Dieudonne, P.; Charnay, C.; Raehm, L.; Durand, J.-O. One-Pot Construction of Multipodal Hybrid Periodic Mesoporous Organosilica Nanoparticles with Crystal-Like Architectures. Adv. Mater. 2015, 27, 145-149. [CrossRef]

35. Teng, Z.; Li, W.; Tang, Y.; Elzatahry, A.; Lu, G.; Zhao, D. Mesoporous Organosilica Hollow Nanoparticles: Synthesis and Applications. Adv. Mater. 2019, 31, 1707612. [CrossRef] 
36. Chen, Y.; Shi, J. Chemistry of Mesoporous Organosilica in Nanotechnology: Molecularly Organic-Inorganic Hybridization into Frameworks. Adv. Mater. 2016, 28, 3235-3272. [CrossRef]

37. Doustkhah, E.; Rostamnia, S.; Imura, M.; Ide, Y.; Mohammadi, S.; Hyland, C.J.T.; You, J.; Tsunoji, N.; Zeynizadeh, B.; Yamauchi, Y. Thiourea Bridged Periodic Mesoporous Organosilica with Ultra-small Pd Nanoparticles for Coupling Reactions. RSC Adv. 2017, 7, 56306-56310. [CrossRef]

38. Vercaemst, C.; Ide, M.; Wiper, P.V.; Jones, J.T.A.; Khimyak, Y.Z.; Verpoort, F. Ethenylene-Bridged Periodic Mesoporous Organosilicas: From E to Z. Chem. Mater. 2009, 21, 5792-5800. [CrossRef]

39. Li, H.; Gascó, C.; Delalande, A.; Charnay, C.; Raehm, L.; Midoux, P.; Pichon, C.; Pleixats, R.; Durand, J.-O. Periodic Mesoporous Organosilica Nanoparticles with BOC Group, towards HIFU Responsive Agents. Molecules 2020, 25, 974. [CrossRef]

40. Hu, X.; Nguyen, K.T.; Jiang, V.C.; Lofland, D.; Moser, H.E.; Pei, D. Macrocyclic Inhibitors for Peptide Deformylase: A Structure-Activity Relationship Study of the Ring Size. J. Med. Chem. 2004, 47, 4941-4949. [CrossRef]

41. Beil, J.B.; Zimmerman, S.C. Synthesis of Nanosized "Cored" Star Polymers. Macromolecules 2004, 37, 778-787. [CrossRef]

42. Dalcanale, E.; Montanari, F. Selective Oxidation of Aldehydes to Carboxylic Acids with Sodium Chlorite-hydrogen Peroxide. J. Org. Chem. 1986, 51, 567-569. [CrossRef]

43. Takeda, K.; Akiyama, A.; Nakamura, H.; Takizawa, S.; Mizuno, Y.; Takayanagi, H.; Harigaya, Y. Dicarbonates: Convenient 4-Dimethylaminopyridine Catalyzed Esterification Reagents. Synthesis 1994, 10, 1063-1066. [CrossRef]

44. Killops, K.L.; Campos, L.M.; Hawker, C.J. Robust, Efficient, and Orthogonal Synthesis of Dendrimers via Thiol-ene "Click" Chemistry. J. Am. Chem. Soc. 2008, 130, 5062-5064. [CrossRef]

45. Sun, F.G.; Li, M.; Gu, Z.H. Pd/norbornene-Catalyzed Sequential ortho-C-H Alkylation and ipso-Alkynylation: A 1,1-dimethyl-2-alkynol Strategy. Org. Chem. Front. 2016, 3, 309-313. [CrossRef]

46. Heathcock, C.H.; Ratcliffe, R. A Stereoselective Total Synthesis of the Guaiazulenic Sesquiterpenoids $\alpha$-Bulnesene and Bulnesol. J. Am. Chem. Soc. 1971, 93, 1746-1757. [CrossRef]

47. Park, S.S.; Jung, M.H.; Lee, Y.S.; Bae, J.H.; Kim, S.H.; Ha, C.S. Functionalised Mesoporous Silica Nanoparticles with Excellent Cytotoxicity against Various Cancer Cells for $\mathrm{pH}$-Responsive and Controlled Drug Delivery. Mater. Des. 2019, 184, 108187. [CrossRef]

48. Xie, M.; Shi, H.; Ma, K.; Shen, H.J.; Li, B.; Shen, S.; Wang, X.S.; Jin, Y. Hybrid Nanoparticles for Drug Delivery and Bioimaging: Mesoporous Silica Nanoparticles Functionalized with Carboxyl Groups and a Near-Infrared Fluorescent Dye. J. Colloid Interface Sci. 2013, 395, 306-314. [CrossRef]

49. Dutta, S.; Kao, H.M.; Wu, K.C.W. Effect of Carboxylic Acid of Periodic Mesoporous Organosilicas on the Fructose-to-5-Hydroxymethylfurfural Conversion in Dimethylsulfoxide Systems. APL Mater. 2014, 2, 113314. [CrossRef]

(C) 2020 by the authors. Licensee MDPI, Basel, Switzerland. This article is an open access article distributed under the terms and conditions of the Creative Commons Attribution (CC BY) license (http://creativecommons.org/licenses/by/4.0/). 